\title{
Neuronal connectivity in major depressive disorder: a systematic review
}

This article was published in the following Dove Press journal:

Neuropsychiatric Disease and Treatment

\section{Katharina Helm ${ }^{1,2}$ \\ Kathrin $\mathrm{Viol}^{3}$ \\ Thomas M Weiger ${ }^{2}$ \\ Peter A Tass ${ }^{4}$ \\ Christian Grefkes ${ }^{5,6}$ \\ Damir del Monte ${ }^{3}$ \\ Günter Schiepek ${ }^{3,7}$}

'Institute of Physiology and

Pathophysiology, Paracelsus Medical

University Salzburg, Salzburg,

Austria; ${ }^{2}$ Department of Biosciences, University of Salzburg, Salzburg,

Austria; ${ }^{3}$ Institute of Synergetics and

Psychotherapy Research, University

Hospital for Psychiatry, Psychotherapy

and Psychosomatics, Paracelsus

Medical University Salzburg,

Salzburg, Austria; ${ }^{4}$ Department of

Neurosurgery, Stanford University,

Stanford CA, USA; ${ }^{5}$ Department

of Neurology, Cologne University

Hospital, Cologne, Germany; ${ }^{6}$ Institute

of Medicine and Neurosciences -

Cognitive Neurology (INM-3),

Research Center Juelich, Juelich,

Germany; ${ }^{7}$ Ludwig Maximilians

University, Department for

Psychology, Munich, Germany
Correspondence: Günter Schiepek Paracelsus Medical University Salzburg, Institute of Synergetics and Psychotherapy Research, University Hospital of Psychiatry, Psychotherapy and Psychosomatics, Ignaz Harrer Str 79, A-5020 Salzburg, Austria

Tel +435725556709

Email guenter.schiepek@ccsys.de
Background: The causes of major depressive disorder (MDD), as one of the most common psychiatric disorders, still remain unclear. Neuroimaging has substantially contributed to understanding the putative neuronal mechanisms underlying depressed mood and motivational as well as cognitive impairments in depressed individuals. In particular, analyses addressing changes in interregional connectivity seem to be a promising approach to capture the effects of MDD at a systems level. However, a plethora of different, sometimes contradicting results have been published so far, making general conclusions difficult. Here we provide a systematic overview about connectivity studies published in the field over the last decade considering different methodological as well as clinical issues.

Methods: A systematic review was conducted extracting neuronal connectivity results from studies published between 2002 and 2015. The findings were summarized in tables and were graphically visualized.

Results: The review supports and summarizes the notion of an altered fronto-limbic mood regulation circuitry in MDD patients, but also stresses the heterogeneity of the findings. The brain regions that are most consistently affected across studies are the orbitomedial prefrontal cortex, anterior cingulate cortex, amygdala, hippocampus, cerebellum and the basal ganglia.

Conclusion: The results on connectivity in MDD are very heterogeneous, partly due to different methods and study designs, but also due to the temporal dynamics of connectivity. While connectivity research is an important step toward a complex systems approach to brain functioning, future research should focus on the dynamics of functional and effective connectivity.

Keywords: major depressive disorder, MDD, structural connectivity, functional connectivity, effective connectivity, fMRI, EEG

\section{Introduction}

Major depressive disorder (MDD) is one of the most common psychiatric diseases. Its major symptoms are depressed mood as well as motivational and cognitive impairments. ${ }^{1-4}$ Even though this psychiatric disorder concerns an increasing number of people and has serious consequences in economic, social, and even political fields, its pathophysiology is still unclear.

The etiology of MDD is ambiguous despite several theoretical approaches and efforts to optimize treatments. One approach is based on the monoamine deficiency hypothesis. Since the catabolic enzymes of drugs like iproniazid or toloxatone influence the serotonergic and catecholaminergic neurotransmission by inhibiting monoamine-oxidase (MAO), these drugs reduce depressive symptoms and operate in a mood-enhancing way. This obvious effect motivated the assumption that a deficiency of neurotransmission by serotonin or catecholamine may contribute to the etiology of MDD. On the one hand, the hypothesis that MDD could be induced by a deficit of monoamines is 
plausible because serotonin-increasing antidepressants have effects and reduce depressive symptoms. On the other hand, this mechanism is questionable because there is a time delay of several weeks in the therapeutic effects of these drugs. If the chemical imbalance of monoamines would be directly linked to symptom severity one should expect a more immediate effect after starting medication since in laboratory experiments the effects on the neurotransmission at the modulatory synapses are instantaneous. ${ }^{5-7}$ Furthermore, not all drugs which increase the pre- or postsynaptic availability of neurotransmitters involved in the regulation of positive emotions are antidepressant. For example, cocaine, which increases norepinephrinergic levels, has no effect on the modulation of mood in MDD. ${ }^{5}$

In consequence, the consistent and scientifically proven virtue of antidepressants might be explained in a different way. Changes in the concentrations of neurotransmitters or neuromodulators could have long-term effects on the neuronal organization of the brain and by this on depressive symptoms. ${ }^{5}$ This requires another theory which might be elaborated using the capabilities of modern functional brain imaging methods. Actually, the concept of pathological neuronal connectivity essentially contributes to the etiological models of MDD and is supported by the fact that antidepressants support the reorganization of pathological connectivity. 8,9

According to this assumption, MDD results more from an information-processing dysfunctionality within neuronal networks than from a chemical imbalance in the brain's molecular architecture. ${ }^{6,10,11}$ Frequently reported in studies focusing on the neuronal network hypothesis are the cortico-limbic network, the default-mode network (DMN), and the affective network. ${ }^{12-15,83}$ The cortico-limbic network is composed of sub-circuits like the fronto-limbic, the parietal-limbic, and the temporo-limbic network. ${ }^{12,15,16}$ The DMN includes the ventromedial prefrontal cortex, the posterior cingulate cortex, and the precunues. ${ }^{13,17-19}$ Functional magnetic resonance imaging (fMRI) studies found that these regions consistently show less activity during stimulus-driven tasks than during resting state. Therefore, these regions could constitute a network supporting a default mode of brain function engaged in introspection and selfreferential processing, like rating the importance of one's own internal and external stimuli of the past and the future. ${ }^{16}$ In this context, other resting-state networks like the salience network ( $\mathrm{SN}$ ) or the central executive network (CEN) seem to be relevant, although the nature of the alterations (hyperor hypo-connectivity) remain inconsistent within these networks and between other brain regions. ${ }^{105}$ In emotion perception and emotion regulation, a network compound of the subgenual and the pregenual anterior cingulate cortex, ie, regions of the limbic system, has close connections to the hypothalamus, the amygdala, and the nucleus accumbens. ${ }^{14,20}$ As both networks are engaged in supervising internal and expressive emotional processing, they are referred to as the introspective socio-affective network (ISA). ${ }^{20}$ In other studies an even greater network is mentioned, the limbic-corticostriato-pallidal-thalamic circuit (LCSPT), which is based on connections between the orbital and medial prefrontal cortex, amygdala, hippocampal subiculum, ventromedial striatum, mediodorsal and midline thalamic nuclei, and the ventral pallidum. ${ }^{21}$ Furthermore, a cerebellar network has been described to be associated with the neuropathology of MDD. ${ }^{13,22}$ Hypoconnectivities within and between all these networks and monitoring systems involved in the perception and the cognitive control of emotions as well as in internal and external attention might contribute to the cognitive and affective abnormalities in depression. ${ }^{16}$

With an increasing number of MDD-related neuroconnectivity studies, the inconsistencies of the results increase. Several reviews and meta-analyses on connectivity in MDD have been published in recent years. These reviews focused on specific questions (eg, alterations during therapy, correlations with specific symptoms), the kind of connectivity (structural, functional, or effective), methods of data acquisition (eg, fMRI, electro-encephalography [EEG], or others), methods of statistical analyses (eg, ICA, seed-based), conditions (eg, resting state or task-related), or on specific subnetworks (eg, the default mode network). ${ }^{105-111}$ To our knowledge, our study is the first to integrate all studies on connectivity in MDD with the aim to consolidate the knowledge on connectivity alterations across methods and conditions.

The studies of our review vary in sample size and stimulation conditions. Also, the anatomical classification of brain regions reported in the studies is inconsistent. In consequence, statistical data analysis is not possible due to the lack of data comparability.

In our systematic review, a fixed scale of 17 brain regions was used to unify the different brain regions reported in the studies under consideration. The aim of this study is to extract and graphically present information from the studies in order to identify valid findings which could motivate future research. Regions of interest should be identified which were involved in altered neuronal connectivity patterns of MDD.

\section{Materials and methods}

A literature search on PubMed (http://www.ncbi.nlm.nih. gov/pubmed) and Embase (http://www.embase.com) was 
conducted for studies published between 2002 and 2015 using the following keywords: "major depression" and "EEG connectivity", "neuronal connectivity" and "major depression" or "major depressive disorder", "connectivity" and "major depression" or "major depressive disorder", "functional connectivity" and "major depression" or "major depressive disorder", "EEG synchronization" and "major depression" or "major depressive disorder", "EEG connectivity" and "major depression" or "major depressive disorder", "fMRI connectivity" and "major depression" or "major depressive disorder". In 2013, we found five times as many studies than in 2005. The total number of studies included in this review is 70, all published in English.

The selected studies were categorized into three groups based on the type of reported connectivity: structural connectivity (SC), functional connectivity (FC), or effective connectivity (EC).

Structural or anatomical connectivity refers to fiber connections between brain areas (the connectome). ${ }^{23}$ Structural networks have been defined by inter-regional correlations of gray matter volume or cortical thickness, and white matter tracking studies used diffusion imaging techniques such as diffusion-tensor imaging (DTI). ${ }^{24}$ Atrophy in gray and white matter tissue leading to alterations in large-scale structural brain networks was found to be associated with MDD. ${ }^{25}$

$\mathrm{FC}$ is defined as the temporal coincidence of spatially distant neurophysiological dynamics. ${ }^{26} \mathrm{FC}$ of two discrete brain regions can be, but may not necessarily be mediated by axons connecting those regions. Numerous studies demonstrated a correlation between structural and FC alterations in MDD, but it is yet not obvious what exactly drives these changes. ${ }^{27,28}$ However, direct structural connections are not necessary to activate two regions in a synchronous manner. It is important to note that FC only reflects correlative interactions. Correlation does not imply any causal relationship between sites or any kind of directionality and is no proof for a direct relation, as correlated activity may be mediated by additional structures or may be induced by third structures as driving forces. ${ }^{11}$

In contrast, EC is defined as the causal influence that one brain area exerts on another. Aertsen and Preiss ${ }^{101}$ proposed that "effective connectivity should be understood as the experiment and time-dependent, simplest possible circuit diagram that would replicate the observed timing relationships between the recorded neurons." Thus EC is more dynamic and can be used to calculate bottom-up (forward) vs top-down (backward) connections between regions. ${ }^{29} \mathrm{EC}$ is usually computed for task-related data, although in principle task-free settings (resting state) can also be analyzed by the connectivity approach. ${ }^{11}$

As a result of this categorization process, six studies on $\mathrm{SC}, 61$ studies on FC (37 fMRI/resting state, $16 \mathrm{fMRI} /$ taskrelated, 4 EEG/resting state, 2 EEG/task-related, 1 magnetoencephalography [MEG]/task-related, 1 single-photon emission computed tomography [SPECT]/task-related), and four studies on EC (2 fMRI/task-related, 1 EEG/task-related, $1 \mathrm{MEG} /$ task-related) were classified. Studies that addressed more than one category or condition were included in both tables with their respective results.

As the nomenclature of the described brain regions in the included studies is inhomogeneous, ranging from very specific regions to whole lobes, a unifying categorization into 17 brain areas was realized (Table 1). Some regions were pooled to greater areas in order to simplify the picture in the graphical analysis. The categorization is based on the brain cartography of Brodmann and Nowinski. ${ }^{30,31}$

Due to many studies with heterogenic results in the categories $\mathrm{fMRI} /$ resting-state and $\mathrm{fMRI} /$ task-related, we provide a tabular and graphical representation of the results for these two categories. The graphical analysis comprises matrices and figures depicting the altered connections between the 17 brain regions. It has to be noted that some studies analyzed the whole brain, whereas others referred to one hemisphere only, or did not provide information about the laterality of the relevant regions. In consequence and for reasons of comparability, all results were projected onto one hemisphere as the frame of reference, ie, the brain was assumed to be symmetrical. It is important to note that this was only done for graphical reasons in order to simplify the resulting pictures. The exact results of all studies are reported in the text as well as in the Tables 2-6.

Furthermore, several studies reported on longitudinal changes before and after therapy. Since the principal interest of this study was not on treatment effects, but to investigate MDD-specific neuronal connectivity, we only used the pretreatment information for the graphical analyses. If a study found an increase of certain connections after therapy compared to the status before treatment, and if this was correlated with psychological improvement, we assumed the decreased connectivity to be pathological. This way, we were able to include also the studies focusing on treatment effects in the graphical representation.

While all studies are listed in the tables, some studies could not be represented in the figures due to the following reasons: some authors mentioned increased or decreased alterations within certain networks without specifying the exact connections within these networks (Veer et $\mathrm{al}^{92}$ 
Table I Coding of brain regions. To reduce the number of all brain regions occurring in the analyzed studies, they were summarized to 17 main regions

\begin{tabular}{|c|c|c|c|}
\hline Code & Color & Brain region & Subregions reported in the studies cited \\
\hline I & $\mathbf{n}$ & Frontal pole & Frontal pole \\
\hline 2 & $\mathbf{m}$ & Orbitofrontal lobe & Medial orbitofrontal cortex, orbitofrontal cortex, orbitomedial prefrontal cortex \\
\hline 3 & $\mathbf{a}$ & Lateral frontal lobe & $\begin{array}{l}\text { Dorsolateral prefrontal cortex, inferior frontal cortex, inferior frontal gyrus, middle } \\
\text { frontal cortex, middle frontal gyrus, prefrontal cortex, rostral PFC, superior frontal } \\
\text { cortex, superior frontal gyrus, subgenual prefrontal cortex, ventrolateral prefrontal } \\
\text { cortex }\end{array}$ \\
\hline 4 & $\mathbf{a}$ & Medial frontal lobe & $\begin{array}{l}\text { Dorsomedial frontal cortex, dorsomedial prefrontal cortex, medial frontal gyrus, } \\
\text { medial prefrontal cortex, medial prefrontal gyrus, superior medial frontal gyrus, } \\
\text { ventromedial frontal cortex, ventromedial prefrontal cortex }\end{array}$ \\
\hline 5 & ש & Sensorimotor (central) & $\begin{array}{l}\text { Paracentral gyrus, paracentral lobule, precentral gyrus, primary sensory motor } \\
\text { cortex, supplementary motor area }\end{array}$ \\
\hline 6 & 口 & Superior parietal lobe & Precuneus, parietal lobe, superior parietal gyrus \\
\hline 7 & - & Inferior parietal lobe & Angular gyrus, inferior parietal cortex, inferior parietal lobe, supramarginal gyrus \\
\hline 8 & - & Temporal pole & Temporal poles \\
\hline 9 & - & Superior temporal lobe & Superior temporal gyrus \\
\hline 10 & $\mathbf{n}$ & Middle temporal lobe & Middle temporal gyrus \\
\hline 11 & $\mathbf{\square}$ & Inferior temporal lobe & Inferior temporal gyrus \\
\hline 12 & $\mathbf{\square}$ & Medial temporal lobe & Amygdala, fusiform gyrus, hippocampus, middle cingulum, parahippocampal gyrus \\
\hline 13 & $\mathbf{\square}$ & Cingulate cortex & $\begin{array}{l}\text { Anterior cingulate cortex, cingulate, dorsal anterior cingulate cortex, pregenual } \\
\text { anterior cingulate cortex, posterior cingulate cortex, rostral anterior cingulate } \\
\text { cortex, subgenual anterior cingulate cortex, subgenual cingulate cortex, supragenual } \\
\text { cingulate cortex }\end{array}$ \\
\hline 14 & $\mathbf{\square}$ & Basal ganglia/thalamus & $\begin{array}{l}\text { Caudate body, claustrum, caudate nucleus, caudate putamen, dorsal caudate, dorsal } \\
\text { caudal putamen, lentiform nucleus, nucleus accumbens, putamen, pallido-striatum, } \\
\text { thalamus, ventral caudate nucleus, ventral rostral putamen, ventral striatum }\end{array}$ \\
\hline 15 & & Insula & $\begin{array}{l}\text { Anterior insula cortex, insula, dorsal insula, dorsal mid insular cortex, fronto insula } \\
\text { operculum, posterior insula }\end{array}$ \\
\hline 16 & ㅁ & Occipital lobe & Cuneus, lingual gyrus, middle occipital gyrus, occipital cortex, superior occipital gyrus \\
\hline 17 & n & Cerebellum & Cerebellum, crus cerebelli, culmen \\
\hline
\end{tabular}

Table 2 Studies on structural connectivity using DTI

\begin{tabular}{|c|c|c|c|c|c|c|c|}
\hline Author & Year & Task & $\begin{array}{l}\text { Decreased } \\
\text { connectivity }\end{array}$ & $\begin{array}{l}\text { Increased } \\
\text { connectivity }\end{array}$ & $\begin{array}{l}\text { Sample } \\
\text { size }\end{array}$ & $\begin{array}{l}\text { Medication } \\
\text { status }\end{array}$ & $\begin{array}{l}\text { Mean } \\
\text { symptom } \\
\text { severity }\end{array}$ \\
\hline Arnold et al' & 2012 & Rest & & $\begin{array}{l}>\text { AMYG-Hc } \\
>\text { AMYG-Ce } \\
>\text { AMYG-BS }\end{array}$ & 17 & Naive & $\begin{array}{l}\text { HDRS } 9.18 \\
\pm 6.82\end{array}$ \\
\hline de Kwaasteniet et $\mathrm{al}^{27}$ & 2013 & Emotional & $<$ subACC-MTL & & 18 & Mixed & $\begin{array}{l}\text { HDRS } 19.17 \\
\pm 3.9\end{array}$ \\
\hline Fang et $\mathrm{al}^{12}$ & 2012 & Cognitive & & $>$ cortico-limbic-NW & 22 & Naive & $\begin{array}{l}\text { HDRS } \\
26.0 \\
\pm 5.1 \\
\end{array}$ \\
\hline Korgaonkar et $\mathrm{al}^{32}$ & 2012 & Rest & \multicolumn{2}{|c|}{$\begin{array}{l}\text { Disparities in white matter tracts of limbic, } \\
\text { frontal, thalamic, cortical connections }\end{array}$} & 23 & Naive & $\begin{array}{l}\text { HDRS 19.1 } \\
\pm 3.0\end{array}$ \\
\hline Singh et $\mathrm{a}^{25}$ & 2013 & Rest & $\begin{array}{l}<\text { Clustering } \\
<\text { Nodes in } \\
\text { medial- frontal/- } \\
\text { temporal regions }\end{array}$ & $>$ AMYG-MeOFG & 93 & Mixed & $\begin{array}{l}\text { BDI } 30.28 \\
\pm 10.17\end{array}$ \\
\hline Xu et $\mathrm{al}^{74}$ & 2013 & Rest & \multicolumn{2}{|c|}{ Anterior genu of corpus callosum altered } & 27 & Naive & HDRS 28.5 \\
\hline
\end{tabular}

Notes: $<$, decreased connectivity; $>$, increased connectivity (if the information was available in the original article). The sample of the study by Arnold et al consisted of patients with remitted MDD.

Abbreviations: ACC, anterior cingulate cortex; AMYG, amygdala; BDI, Beck Depression Inventory; BS, brain stem; Ce, cerebellum; DTI, diffusion-tensor imaging; Hc, hippocampus; HDRS, Hamilton Depression Rating Scale; MeOFG, medial orbitofrontal gyrus; MTL, middle temporal lobe; NW, network. 
Table 3 Studies on functional connectivity with $\mathrm{fMRI}$ in resting state

\begin{tabular}{|c|c|c|c|c|c|c|}
\hline Author & Year & $\begin{array}{l}\text { Decreased } \\
\text { connectivity }\end{array}$ & $\begin{array}{l}\text { Increased } \\
\text { connectivity }\end{array}$ & $\begin{array}{l}\text { Sample } \\
\text { size }\end{array}$ & $\begin{array}{l}\text { Medication } \\
\text { status }\end{array}$ & $\begin{array}{l}\text { Mean symptom } \\
\text { severity }\end{array}$ \\
\hline Abbott et al ${ }^{85}$ & 2013 & \multicolumn{2}{|c|}{ ECT-therapy normalized DMN-PFC in responders } & 9 & Medicated & $\begin{array}{l}\text { HDRS } \\
\text { pre-treatment } \\
\text { (responders) } 34.6\end{array}$ \\
\hline Anand et $\mathrm{a}^{164}$ & 2005 & $\begin{array}{l}<\text { ACC-PST } \\
<\text { ACC-MeTHAL }\end{array}$ & & 15 & Naive & $\begin{array}{l}\text { HDRS } \\
31 \\
\pm 8\end{array}$ \\
\hline Cao et $\mathrm{al}^{44}$ & 2012 & & $\begin{array}{l}>\text { IHc-MFG } \\
>\text { rHc-rIPC } \\
>\text { rHc-rCe }\end{array}$ & 42 & Naive & $\begin{array}{l}\text { HDRS } \\
23.60(3.77)\end{array}$ \\
\hline Chen et al ${ }^{17}$ & 2015 & $\begin{array}{l}<\text { PCC-DMePFC } \\
<\text { PCC-rIPG } \\
<\text { rTHAL-Ce }\end{array}$ & & 38 & Naive & $\begin{array}{l}\text { HDRS } \\
21.1 \\
\pm 6.5\end{array}$ \\
\hline Connolly et al ${ }^{46}$ & 2013 & $\begin{array}{l}<\text { subACC PCu } \\
<\text { subACC MFG } \\
<\text { subACC-IFG }\end{array}$ & $\begin{array}{l}>\text { subACC-Ci } \\
>\text { subACC-AMYG }\end{array}$ & 23 & Naive & $\begin{array}{l}\text { BDI } \\
27.3 \\
\pm 2.1 \\
\end{array}$ \\
\hline Cullen et $\mathrm{a}^{34}$ & 2009 & $\begin{array}{l}<\text { subACC-supACC } \\
<\text { subACC-rMFC } \\
<\text { subACC-ISFC } \\
<\text { subACC-IIFC } \\
<\text { subACC-ISTG } \\
<\text { subACC-Ci }\end{array}$ & & 12 & Mixed & $\begin{array}{l}\text { BDI } \\
27.7 \\
\pm 11.3\end{array}$ \\
\hline Davey et $\mathrm{a}{ }^{47}$ & 2012 & $<\mathrm{pACC}-\mathrm{CN}$ & $\begin{array}{l}>\text { subACC-DMeFC } \\
>\text { pACC-IDLPFC } \\
>\text { subACC-MFG } \\
>\text { pACC-MFG }\end{array}$ & 18 & Mixed & $\begin{array}{l}\text { BDI } \\
35.4\end{array}$ \\
\hline Downar et $\mathrm{al}^{22}$ & 2014 & \multicolumn{2}{|c|}{$\begin{array}{l}\text { Comparison of TMS responders to non-responders: } \\
\text { reward pathway }\end{array}$} & 47 & Naive & $\begin{array}{l}\mathrm{BDI} \\
32.6\end{array}$ \\
\hline Fox et $\mathrm{al}^{49}$ & 2012 & & $\begin{array}{l}\text { TMS treatment outcome: } \\
\text { > DLPFC-subCC }\end{array}$ & 13 & Mixed & $\begin{array}{l}\text { HDRS } \\
23.8\end{array}$ \\
\hline Furman et $\mathrm{al}^{52}$ & 2011 & $\begin{array}{l}<\text { VS-IsubACC } \\
<\text { VS-MeOFC } \\
<\text { DC-IMeFG } \\
<\text { VRP-ISTG } \\
<\text { VRP-IMeFG } \\
<\text { VRP-OFC } \\
<\text { VRP-rPCi } \\
<\text { DCP-ISTG } \\
<\text { DCP-rSTG } \\
<\text { DCP-rPCi } \\
<\text { DCP-rMTG }\end{array}$ & $\begin{array}{l}>\text { VS-IPCu } \\
>\text { VS-PCC } \\
>\text { VS-rCe } \\
>\text { VS-rIPL } \\
>\text { DC-rMFG } \\
>\text { VRP-rCB } \\
>\text { DCP-IIPL } \\
>\text { DCP-rIPL } \\
>\text { DCP-rLG }\end{array}$ & 21 & Mixed & $\begin{array}{l}\mathrm{BDI} \\
31.3\end{array}$ \\
\hline Greicius et $\mathrm{al}^{51}$ & 2007 & & $\begin{array}{l}\text { > subCC-DMN (MePFC, } \\
\text { ACC) } \\
>\text { THAL-DMN (MePFC, } \\
\text { ACC) }\end{array}$ & 28 & Mixed & $\begin{array}{l}\text { HDRS } \\
25.4 \\
\pm 4\end{array}$ \\
\hline Guo et al ${ }^{19}$ & 2013 & $\begin{array}{l}<\text { IPCC-ITHA } \\
<\text { IPCC-rTHA } \\
<\text { IPCC-rSMeFG } \\
<\text { IPCu-ITHA } \\
<\text { IPCu-rTHA } \\
<\text { IPCu-rSMeFG } \\
>\text { IPCC/IPCu-IIFG } \\
<\text { rPCC/rPCu-ISFG } \\
<\text { rPCC/rPCu-ITHA }\end{array}$ & & 24 & Naive & $\begin{array}{l}\text { HDRS } \\
25.75 \\
\pm 5.86\end{array}$ \\
\hline
\end{tabular}


Table 3 (Continued)

\begin{tabular}{|c|c|c|c|c|c|c|}
\hline Author & Year & $\begin{array}{l}\text { Decreased } \\
\text { connectivity }\end{array}$ & $\begin{array}{l}\text { Increased } \\
\text { connectivity }\end{array}$ & $\begin{array}{l}\text { Sample } \\
\text { size }\end{array}$ & $\begin{array}{l}\text { Medication } \\
\text { status }\end{array}$ & $\begin{array}{l}\text { Mean symptom } \\
\text { severity }\end{array}$ \\
\hline & & $\begin{array}{l}<\text { IMePFC-rMFG } \\
<\text { IMePFC-IMFG } \\
<\text { IMePFC-rSFG } \\
<\text { rMePFC-ISFG } \\
<\text { rMePFC-rACC }\end{array}$ & & & & \\
\hline Guo et $\mathrm{al}^{86}$ & 2013 & $\begin{array}{l}<\text { Ce-PFC (TRD and TSD) } \\
<\text { Ce-SFG (TRD and TSD) } \\
<\text { Ce-IFG (TRD and TSD) } \\
<\text { Ce-MFG (TRD and TSD) } \\
<\text { Ce-DMN (STG, MTG, ITG, } \\
\text { PCu, IPL) (TRD and TSD; } \\
\text { more decreased for TRD) }\end{array}$ & $\begin{array}{l}>\text { Ce-LG (TRD and TSD) } \\
>\text { Ce-MOG (TRD and } \\
\text { TSD) } \\
>\text { Ce-FG (TRD and TSD) } \\
>\text { Ce-PHG (TRD and } \\
\text { TSD) }\end{array}$ & $\begin{array}{l}45 \text { (23 TRD, } \\
22 \text { TSD) }\end{array}$ & Medicated & $\begin{array}{l}\text { HDRS } \\
\text { TRD: } 24.5 \\
\text { TSD: } 25.9\end{array}$ \\
\hline Guo et $\mathrm{al}^{2}$ & 2013 & $\begin{array}{l}<\text { ICalc-IITG (TRD) } \\
<\text { ICalc-rLG (TRD) } \\
<\text { ICalc-rPoG (TRD) } \\
<\text { ICalc-ISTG (TRD) } \\
<\text { ICalc-IPCu (TRD) } \\
<\text { ICalc-rCalc (TRD) } \\
<\text { ICalc-rMTG (TRD) } \\
<\text { ICalc-IPG (TRD) } \\
<\text { rCalc-I/rMTG (TRD and TSD) } \\
<\text { rCalc-ILG (TRD) } \\
<\text { rCalc-rMOG (TRD) } \\
<\text { rCalc-rTHAL (TRD) } \\
<\text { rCalc-IPG (TRD) } \\
<\text { rCalc-rPoG (TRD) }\end{array}$ & & $\begin{array}{l}45 \text { (23 TRD, } \\
22 \text { TSD) }\end{array}$ & Mixed & $\begin{array}{l}\text { HDRS } \\
\text { TRD: } 24.5 \\
\text { TSD: } 25.9\end{array}$ \\
\hline Guo et $\mathrm{al}^{87}$ & 2015 & $\begin{array}{l}<\text { rCi-IMFG } \\
<\text { rCi-ISTG } \\
<\text { rCi-rP } \\
<\text { rCi-rMOG } \\
<\text { ICi-ISTP } \\
<\text { ICi-rMOG }\end{array}$ & & 44 & Naive & $\begin{array}{l}\text { HDRS } \\
25.2 \\
\pm 5.2\end{array}$ \\
\hline Horn et $\mathrm{al}^{3}$ & 2010 & & $>$ pACC-ACi & 28 & Medicated & $\begin{array}{l}\text { HDRS } \\
17.2 \\
\pm 4.7 \\
\end{array}$ \\
\hline Kozel et al ${ }^{8}$ & 2011 & \multicolumn{2}{|c|}{ AD treatment outcome highly related with SCaIC } & 13 & Medicated & $\begin{array}{l}\text { HDRS not } \\
\text { available }\end{array}$ \\
\hline Lai et $a^{88}$ & 2014 & $\begin{array}{l}<\text { Within MFC } \\
<\text { Within ACC } \\
<\text { Within Ce }\end{array}$ & & 44 & Naive & $\begin{array}{l}\text { HDRS } \\
22.1 \\
\pm 2.3 \\
\end{array}$ \\
\hline Li et al ${ }^{9}$ & 2013 & \multicolumn{2}{|c|}{$\begin{array}{l}>\mathrm{aDMN} \text { und }>\mathrm{pDMN} \text { before AD treatment, normalized } \\
\text { after treatment }\end{array}$} & 24 & Medicated & $\begin{array}{l}\text { HDRS } \\
26.42 \\
\pm 5.22 \\
\end{array}$ \\
\hline Liu et a ${ }^{89}$ & 2012 & $\begin{array}{l}<\text { Ce-DMN } \\
(\text { VMPFC, PCC, PCu) } \\
<\text { Ce-PCu } \\
<\text { Ce-FG } \\
<\text { Ce-IOFC } \\
<\text { Ce-SFG } \\
<\text { Ce-rSTG } \\
<\text { Ce-rLG } \\
<\text { Ce-HC }\end{array}$ & $\begin{array}{l}>\text { Ce-TP } \\
>\text { Ce-IMFC } \\
>\text { Ce-rITG } \\
>\text { Ce-ISTG }\end{array}$ & 60 & Naive & $\begin{array}{l}\text { HDRS } \\
26.1 \\
\pm 5.0\end{array}$ \\
\hline Ma et $\mathrm{a}^{28}$ & 2012 & $\begin{array}{l}<\text { rMTG-rAG (TSD) } \\
<\text { rMTG-IPCu (TSD) } \\
<\text { rMTG-IPHG (TSD) } \\
<\text { rMTG-rCu (TRD) }\end{array}$ & $\begin{array}{l}>\text { rMTG-ISMG (TSD) } \\
>\text { rMTG-rPCu (TRD) } \\
>\text { within rMTG (TRD) }\end{array}$ & $\begin{array}{l}35 \text { (I8 TRD, } \\
17 \text { TSD) }\end{array}$ & Mixed & $\begin{array}{l}\text { HDRS } \\
\text { TRD: } 23.9 \\
\text { TSD: } 25.6\end{array}$ \\
\hline
\end{tabular}


Table 3 (Continued)

\begin{tabular}{|c|c|c|c|c|c|c|}
\hline Author & Year & $\begin{array}{l}\text { Decreased } \\
\text { connectivity }\end{array}$ & $\begin{array}{l}\text { Increased } \\
\text { connectivity }\end{array}$ & $\begin{array}{l}\text { Sample } \\
\text { size }\end{array}$ & $\begin{array}{l}\text { Medication } \\
\text { status }\end{array}$ & $\begin{array}{l}\text { Mean symptom } \\
\text { severity }\end{array}$ \\
\hline & & $\begin{array}{l}<\text { rCau-rMFG }(\text { TSD) } \\
<\text { rCau-rIFG }(\text { TSD) } \\
<\text { rCau-rCi }(T S D) \\
<\text { rCau-IMOG }(\text { TRD + TSD) } \\
<\text { rCau-rOFC }(\text { TRD })\end{array}$ & $\begin{array}{l}>\text { rMTG-I/r SFG (TRD) } \\
>\text { rMTG-IMFG (TRD) } \\
>\text { rCau-rIFG (TSD) } \\
>\text { rCau-rMFG (TSD) } \\
>\text { rCau-I/r SFG (TSD) }\end{array}$ & & & \\
\hline Perrin et a $9^{90}$ & 2012 & \multicolumn{2}{|c|}{ Electroconvulsive therapy outcome: $<$ within IDLPFC } & 9 & Medicated & $\begin{array}{l}\text { MADRS } \\
36.4 \\
\pm 4.9 \\
\end{array}$ \\
\hline $\begin{array}{l}\text { Ramasubbu } \\
\text { et } \mathrm{al}^{91}\end{array}$ & 2014 & $\begin{array}{l}<\text { AMYG-VLPFC } \\
<\text { AMYG-Ci } \\
<\text { AMYG-CP } \\
<\text { AMYG-MTG } \\
<\text { AMYG-STG } \\
<\text { AMYG }{ }^{\circ} \\
<\text { AMYG-Ce }\end{array}$ & & 55 & Naive & $\begin{array}{l}\text { HDRS } 2 \mathrm{I} .4 \mathrm{I} \\
\pm 2.2\end{array}$ \\
\hline Salomons et al ${ }^{4}$ & 2014 & \multicolumn{2}{|c|}{$\begin{array}{l}\text { Successful TMS treatment associated with } \\
>\text { DMePFC-subCC } \\
>\text { subCC-DLPFC } \\
<\text { cortico-THAL } \\
<\text { cortico-striatal } \\
<\text { cortico-limbic } \\
>\text { DMePFC-THAL } \\
<\text { subCC-Cau }\end{array}$} & 25 & Mixed & $\begin{array}{l}\text { HDRS } 21.3 \\
\pm 6.7\end{array}$ \\
\hline Sheline et $\mathrm{al}^{14}$ & 2010 & & $\begin{array}{l}>\text { DLPFC-DMPFC } \\
>\text { Pcu-DMPFC } \\
>\text { subACC-DMPFC }\end{array}$ & 18 & Naive & $\begin{array}{l}\text { HDRS } 20.2 \\
\pm 2.9\end{array}$ \\
\hline $\begin{array}{l}\text { Tahmasian et } \\
\mathrm{al}^{45}\end{array}$ & 2013 & $\begin{array}{l}<\text { AMYG-rMeFG } \\
<\text { AMYG-rSFG } \\
<\text { AMYG-rFIO } \\
<\text { AMYG-rIFG } \\
<\text { AMYG-IIPL } \\
<\text { Hc-rSFG } \\
<\text { Hc-rFIO } \\
<\text { Hc-IIFG } \\
<\text { Hc-IMeFG } \\
<\text { Hc-IMFG } \\
<\text { Hc-rIPL }\end{array}$ & & 21 & Medicated & $\begin{array}{l}\text { BDI } 25.3 \\
\pm 7.1\end{array}$ \\
\hline Veer et $\mathrm{al}^{92}$ & 2010 & $\begin{array}{l}<\text { Within IFP } \\
<\text { Within LG }\end{array}$ & $\begin{array}{l}>\text { Within affective } \\
\text { network (AMYG, } \\
\text { IACi) }\end{array}$ & 19 & Naive & $\begin{array}{l}\text { MADRS I4.2I } \\
\pm 9.62\end{array}$ \\
\hline Wang et a ${ }^{93}$ & 2014 & $\begin{array}{l}<\text { Within VMPFC } \\
<\text { Within VACC }\end{array}$ & & 20 & Mixed & HDRS 27.I \\
\hline Wang et a ${ }^{94}$ & 2013 & $\begin{array}{l}<\text { Within MeOFC } \\
<\text { Within PHG } \\
<\text { Within FG } \\
<\text { Within MOG } \\
<\text { Within } \mathrm{Cu}\end{array}$ & & 17 & Naive & $\begin{array}{l}\text { HDRS } 26.58 \\
\pm 3.43\end{array}$ \\
\hline Wei et al ${ }^{95}$ & 2013 & $\begin{array}{l}<\text { rfronto-parietal NW } \\
<\text { DMN }\end{array}$ & $\begin{array}{l}\text { > Ifronto-parietal } \\
\text { NW (IFG, MFG, } \\
\text { PCu, IPG, AG) } \\
\text { > ventromedial- } \\
\text { prefrontal NW } \\
\text { > salience NW } \\
\text { (ACC, aCi, MFG) }\end{array}$ & 20 & Naive & $\begin{array}{l}\text { HDRS } 25.8 \\
\pm 2.4\end{array}$ \\
\hline
\end{tabular}

(Continued) 
Table 3 (Continued)

\begin{tabular}{|c|c|c|c|c|c|c|}
\hline Author & Year & $\begin{array}{l}\text { Decreased } \\
\text { connectivity }\end{array}$ & $\begin{array}{l}\text { Increased } \\
\text { connectivity }\end{array}$ & $\begin{array}{l}\text { Sample } \\
\text { size }\end{array}$ & $\begin{array}{l}\text { Medication } \\
\text { status }\end{array}$ & $\begin{array}{l}\text { Mean symptom } \\
\text { severity }\end{array}$ \\
\hline Ye et $a^{50}$ & 2012 & $<$ rDLPFC-rPL & $\begin{array}{l}>\text { rDLPFC-IACC } \\
>\text { rDLPFC-IPHG } \\
>\text { rDLPFC-THAL } \\
>\text { rDLPFC-PG }\end{array}$ & 22 & Mixed & HDRS 18.5 \\
\hline Yu et al ${ }^{48}$ & 2013 & \multicolumn{2}{|c|}{$\begin{array}{c}\text { Disciriminative power for: } \\
\text { MePFC-PCu } \\
\text { MePFC-THAL } \\
\text { MePFC-ITG } \\
\text { THAL-ACC } \\
\text { THAL-Hc } \\
\text { Ce-MePFC } \\
\text { Ce-Hc } \\
\text { Ce-THAL } \\
\text { Ce-ACC }\end{array}$} & 19 & - & $\begin{array}{l}\text { HDRS } 25.43 \\
\pm 6.34\end{array}$ \\
\hline Zeng et $\mathrm{al}^{15}$ & 2012 & & $\begin{array}{l}\text { AMYG-PFC } \\
\text { AMYG-VC } \\
\text { AMYG-Ce } \\
\text { PHG-ITG } \\
\text { PHG-STP } \\
\text { PHG-ACC } \\
\text { PHG-PCC } \\
\text { Hc-PFC } \\
\text { Hc-IOG } \\
\text { Hc-AMYG } \\
\text { Hc-Ce }\end{array}$ & 24 & Naive & $\begin{array}{l}\text { HDRS } 26.42 \\
\pm 5.22\end{array}$ \\
\hline Zeng et $\mathrm{a}^{65}$ & 2014 & & $\begin{array}{l}\text { sACC-VLPFC sACC- } \\
\text { VMePFC sACC-STG } \\
\text { sACC-Lnuc sACC-Hc } \\
\text { sACC-Ci sACC-THAL } \\
\text { sACC-SFG } \\
\text { sACC-AMYG pACC- } \\
\text { MePFC pACC-Pcu } \\
\text { PACC-SFG pACC-Ci } \\
\text { PACC-OFC pACC-MTG } \\
\text { PACC-STG } \\
\text { PACC-THAL } \\
\text { PACC-AG pACC-ITG } \\
\text { PACC-SMG } \\
\text { PACC-P }\end{array}$ & 24 & Naive & $\begin{array}{l}\text { HDRS } 26.42 \\
\pm 5.22\end{array}$ \\
\hline Zhang et $\mathrm{al}^{96}$ & 2015 & $\begin{array}{l}<\text { PCC-MTG } \\
<\text { PCC-Pcu }\end{array}$ & $\begin{array}{l}>\text { PCC-MFG } \\
>\text { PCC-SFG }\end{array}$ & 45 & Naive & $\begin{array}{l}\text { HDRS } \\
27.3 \\
\pm 7\end{array}$ \\
\hline Zhang97 & 2011 & & centralities & 30 & Naive & $\begin{array}{l}\text { HDRS } 24.3 \\
\pm 5.0\end{array}$ \\
\hline Zhu ${ }^{98}$ & 2012 & $\begin{array}{l}<\text { Within PCC } \\
<\text { Within Pcu }\end{array}$ & $\begin{array}{l}>\text { Within MePFC } \\
>\text { Within ACC }\end{array}$ & 35 & Naive & $\begin{array}{l}\text { CES-D } 38.03 \\
\pm 6.67\end{array}$ \\
\hline
\end{tabular}

Notes: $<$, decreased connectivity; $>$, increased connectivity (if the information was available in the original article). A small $r$ or I in front of the regions indicates if the finding was in the left $(I)$ or right $(r)$ hemisphere.

Abbreviations: $\mathrm{ACC}$, anterior cingulate cortex; $\mathrm{ACi}$, anterior $\mathrm{Ci}$; aDMN, anterior DMN; $\mathrm{AG}$, angular gyrus; $\mathrm{BDI}$, Beck Depression Inventory; Calc, calcarine cortex; Cau, caudatus; CB, caudate body; CC, cingulate cortex; Ce, cerebellum; CES-D, Center For Epidemiologic Studies Depression Scale; Ci, insula; CN, caudate nucleus; CP, caudate putamen; Cu, cuneus; DC, dorsal caudate; DLPFC, dorsolateral PFC; DMePFC, dorsal medial PFC; DMN, default mode network; FG, fusiform gyrus; FIO, fronto insula operculum; fMRI, functional magnetic resonance imaging; FP, frontal pole; Hc, hippocampus; HDRS, Hamilton Depression Rating Scale; IFG, inferior frontal gyrus; IOG, inferior occipital gyrus; IPL, inferior parietal lobe; ITG, inferior temporal gyrus; LG, lingual gyrus; Lnuc, lentiform nucleus; MADRS, Montgomery-Asberger Depression Rating Scale; MC, middle cingulum; MeFG, medial frontal gyrus; MeOFC, medial OFC; MePFC, medial PFC; MFC, middle frontal cortex; MFG, middle frontal gyrus; MOG, middle occipital gyrus; MTG, middle temporal gyrus; OC, occipital cortex; OFC, orbitofrontal cortex; P, putamen; PACC, pregenual ACC; PCC, posterior cingulate cortex; PCG, paracentral gyrus; PCu, precuneus; pDMN, posterior DMN; PFC, prefrontal cortex; PG, precentral gyrus; PHG, parahippocampus; PL, parietal lobe; PST, pallido-striatum; sACC, subgenual ACC; SCalC, subcallosal cortex; subCC, subgenual CC; SFG, superior frontal gyrus; SMG, supramarginal gyrus; STG, superior temporal gyrus; STP, superior temporal pole; TP, temporal poles; TRD, treatment-resistant depression; TSD, treatment-sensitive depression; VACC, ventral ACC; VC, visual cortex; VLPFC, ventrolateral PFC; VMePFC, ventromedial PFC; VRP, ventral rostral putamen; vs, ventral striatum. 
Table 4 Studies on functional connectivity in task-related fMRI

\begin{tabular}{|c|c|c|c|c|c|c|c|}
\hline Author & Year & Task & Decreased connectivity & $\begin{array}{l}\text { Increased } \\
\text { connectivity }\end{array}$ & $\begin{array}{l}\text { Sample } \\
\text { size }\end{array}$ & $\begin{array}{l}\text { Medication } \\
\text { status }\end{array}$ & $\begin{array}{l}\text { Mean } \\
\text { symptom } \\
\text { severity }\end{array}$ \\
\hline Anand ${ }^{64}$ & 2005 & $\begin{array}{l}\text { Emotional } \\
\text { pictures }\end{array}$ & $\begin{array}{l}<\text { ACC-PST } \\
<\text { ACC-MeTHAL }\end{array}$ & & 15 & Naive & $\begin{array}{l}\text { HDRS } \\
31 \\
\pm 8\end{array}$ \\
\hline Avery et $\mathrm{a}^{56}$ & 2014 & $\begin{array}{l}\text { Visceral } \\
\text { interoceptive } \\
\text { sensation }\end{array}$ & & $\begin{array}{l}>\text { IDMCi-rMTG } \\
>\text { IDMCi-IAMYG } \\
>\text { IDMCi-rMOG } \\
>\text { IDMCi-IMeOFC } \\
>\text { IDMCi-rMeOFC } \\
>\text { IDMCi-rCe } \\
>\text { rDMCi-IMeOFC } \\
>\text { rDMCi-rMeOFC } \\
>\text { rDMCi-rMTG } \\
>\text { rDMCi-rMOG } \\
>\text { rDMCi-IPG } \\
>\text { rDMCi-IAMYG } \\
>\text { rDMCi-IOFC }\end{array}$ & 20 & Naive & $\begin{array}{l}\text { HDRS } \\
23.1 \\
\pm 7.5\end{array}$ \\
\hline Henje Blom et al99 & 2015 & $\begin{array}{l}\text { Emotional } \\
\text { face }\end{array}$ & & $\begin{array}{l}>\text { ACi-rFG } \\
>\text { ACi-IMFG } \\
>\text { ACi-rAMYG } \\
>\text { ACi-PHG }\end{array}$ & 31 & Naive & $\begin{array}{l}\text { BDI } \\
29.1 \\
\pm 1.7\end{array}$ \\
\hline Davey et $\mathrm{a}^{54}$ & 2012 & $\begin{array}{l}\text { Executive } \\
\text { control }\end{array}$ & $<$ subACC-MFG & $>$ subACC-rVS & 17 & Mixed & $\begin{array}{l}\text { BDI } \\
34.4 \\
\pm 12.6\end{array}$ \\
\hline $\begin{array}{l}\text { de Kwaasteniet } \\
\text { et } \mathrm{al}^{27}\end{array}$ & 2013 & $\begin{array}{l}\text { Emotional } \\
\text { face }\end{array}$ & & $>$ subACC-MTL & 18 & mixed & $\begin{array}{l}\text { HDRS } \\
19.17 \\
\pm 3.9\end{array}$ \\
\hline Frodl et $\mathrm{a}^{53}$ & 2010 & $\begin{array}{l}\text { Emotional } \\
\text { face }\end{array}$ & & $\begin{array}{l}>\text { OFC-rPCu } \\
>\text { OFC-ICe } \\
>\text { OFC-IMC } \\
>\text { OFC-ISOG } \\
>\text { OFC-rCrC } \\
>\text { OFC-rMTG } \\
>\text { OFC-rCe } \\
>\text { OFC-rSPG } \\
>\text { OFC-rTHAL }\end{array}$ & 25 & Naive & $\begin{array}{l}\text { HDRS } \\
20.6 \\
\pm 5.2\end{array}$ \\
\hline Hamilton et al ${ }^{58}$ & 2008 & $\begin{array}{l}\text { Emotional } \\
\text { pictures }\end{array}$ & & $\begin{array}{l}>\text { rAMYG-Hc } \\
>\text { rAMYG-CP }\end{array}$ & 14 & Mixed & $\begin{array}{l}\text { BDI } \\
27.6 \\
\pm 10.6\end{array}$ \\
\hline Heller et $\mathrm{a}^{62}$ & 2013 & $\begin{array}{l}\text { Emotional } \\
\text { pictures }\end{array}$ & \multicolumn{2}{|c|}{$\begin{array}{c}>\text { Fronto-striatal-connectivity is correlated with } \\
\text { positive treatment effect }\end{array}$} & 21 & Medicated & $\begin{array}{l}\text { HDRS } \\
20.6 \\
\pm 2.39\end{array}$ \\
\hline Ho et $a^{55}$ & 2014 & $\begin{array}{l}\text { Emotional } \\
\text { face }\end{array}$ & $\begin{array}{l}<\text { rACC-IFG } \\
<\text { rACC-rPCu } \\
<\text { rACC-ICi } \\
<\text { rACC-rMFG } \\
<\text { rACC-IC } \\
<\text { rACC-rSTG } \\
<\text { rACC-rMTG } \\
<\text { IACC-ICi } \\
<\text { IACC-IC } \\
<\text { IACC-rCi } \\
<\text { IACC-IMFG } \\
<\text { IACC-IMTG }\end{array}$ & $>$ rACC-IAMYG & 19 & Naive & $\begin{array}{l}\text { BDI } \\
23.05 \\
\pm 2.6\end{array}$ \\
\hline
\end{tabular}

(Continued) 
Table 4 (Continued)

\begin{tabular}{|c|c|c|c|c|c|c|c|}
\hline Author & Year & Task & Decreased connectivity & $\begin{array}{l}\text { Increased } \\
\text { connectivity }\end{array}$ & $\begin{array}{l}\text { Sample } \\
\text { size }\end{array}$ & $\begin{array}{l}\text { Medication } \\
\text { status }\end{array}$ & $\begin{array}{l}\text { Mean } \\
\text { symptom } \\
\text { severity }\end{array}$ \\
\hline Kong et al ${ }^{100}$ & 2013 & $\begin{array}{l}\text { Emotional } \\
\text { face }\end{array}$ & $<$ AMYG-IRPFC & & 28 & Naive & $\begin{array}{l}\text { HDRS } \\
28.5 \\
\pm 5.2\end{array}$ \\
\hline Lemogne et al ${ }^{59}$ & 2009 & $\begin{array}{l}\text { Personality } \\
\text { traits }\end{array}$ & & $\begin{array}{l}>\text { MFG-DACC } \\
>\text { MFG-DLPFC }\end{array}$ & 15 & Medicated & $\begin{array}{l}\text { BDI } \\
\text { I8.4 }\end{array}$ \\
\hline Marchand et $\mathrm{a}^{63}$ & 2013 & $\begin{array}{l}\text { Executive } \\
\text { control }\end{array}$ & & $\begin{array}{l}\text { Unipolar vs } \\
\text { bipolar: } \\
\text { > PCC-IACC } \\
>\text { PCC-IMeFG } \\
>\text { PCC-I+ rCi } \\
>\text { PCC-I+ rIFG } \\
>\text { PCC-I+ rMFG } \\
>\text { PCC-ISFG }\end{array}$ & 26 & Naive & $\begin{array}{l}\text { MADRS } \\
26.6 \\
\pm 4.0\end{array}$ \\
\hline Marchand et $\mathrm{al}^{57}$ & 2012 & $\begin{array}{l}\text { Executive } \\
\text { control }\end{array}$ & $\begin{array}{l}<\text { P-IFG } \\
<\text { P-SMA } \\
<\text { P-ACC } \\
<\text { P-SI/MI } \\
<\text { P-THAL } \\
<\text { THAL-IFG } \\
<\text { THAL-SMA } \\
<\text { THAL-ACC } \\
<\text { THAL-SI/MI }\end{array}$ & & 18 & Naive & $\begin{array}{l}\text { MADRS } \\
26.3\end{array}$ \\
\hline Strigo et al ${ }^{102}$ & 2013 & $\begin{array}{l}\text { Pain } \\
\text { anticipation }\end{array}$ & & $\begin{array}{l}>\text { DCi-PTHAL } \\
<\text { Dci-rIFG }\end{array}$ & 31 & Naive & $\begin{array}{l}\mathrm{BDI} \\
2.5\end{array}$ \\
\hline $\operatorname{van}$ Tol et $\mathrm{al}^{60}$ & 2013 & $\begin{array}{l}\text { Emotional } \\
\text { words }\end{array}$ & $\begin{array}{l}<\text { FONW-rOFC } \\
<\text { FONW-INA } \\
<\text { FONW-rMePFG } \\
<\text { FONW-rFP } \\
<\text { FONW-IMePFG } \\
<\text { FONW-rVCN } \\
<\text { FONW-rP } \\
<\text { FONW-rACC }\end{array}$ & & 25 & Naive & $\begin{array}{l}\text { MADRS } \\
14.4 \\
\pm 10.2\end{array}$ \\
\hline Vasic et $\mathrm{al}^{61}$ & 2009 & $\begin{array}{l}\text { Working } \\
\text { memory }\end{array}$ & $\begin{array}{l}\text { FC within NW: } \\
<\text { inferior-parietal regions } \\
<\text { superior prefrontal regions } \\
<\text { frontopolar regions }\end{array}$ & $\begin{array}{l}>\text { IDLPFC } \\
>\text { Ce } \\
>\text { ACC } \\
>\text { VLPFC } \\
>\text { SPFC }\end{array}$ & 14 & Medicated & $\begin{array}{l}\text { BDI } \\
20.5 \\
\pm 8.8\end{array}$ \\
\hline
\end{tabular}

Notes: $<$, decreased connectivity; $>$, increased connectivity (if the information was available in the original article). A small $r$ or I in front of the regions indicates if the finding was on the left or right hemisphere.

Abbreviations: ACC, anterior cingulate cortex; $\mathrm{ACi}$, anterior $\mathrm{Ci}$; BDI, Beck Depression Inventory; C, cingulate; Ce, cerebellum; Ci, insula; CN, caudate nucleus; CP, caudate putamen; CrC, crus cerebelli; DACC, dorsal ACC; DCi, dorsal Ci; DLPFC, dorsolateral PFC; DMCi, dorsal mid-insular cortex; FG, fusiform gyrus; FONW, frontoopercular network; fMRI, functional magnetic resonance imaging; FP, frontal pole; Hc, hippocampus; HDRS, Hamilton Depression Rating Scale; IFG, inferior frontal gyrus; IPG, inferior parietal gyrus; MADRS, Montgomery-Asberger Depression Rating Scale; MC, middle cingulum; MeFG, medial frontal gyrus; MeOFC, medial OFC; MePFC, medial PFC; MeThal, medial Thalamus; MFG, middle frontal gyrus; MOG, middle occipital gyrus; MTG, middle temporal gyrus; NA, nucleus accumbens; OFC, orbitofrontal cortex; P, putamen; PCC, posterior cingulate cortex; PCu, precuneus; PFC, prefrontal cortex; PG, precentral gyrus; PHG, parahippocampus; PST, pallidostriatum; RPFC, rostral PFC; SI/MI, primary sensorimotor cortex; SFG, superior frontal gyrus; SMA, suppl. motor area; SOG, superior occipital gyrus; SPFC, superior PFC; SPG, superior parietal gyrus; STG, superior temporal gyrus; subACC, subgenual ACC; VCN, ventral CN; VLPFC, ventrolateral PFC; VMePFC, ventromedial PFC; vs, ventral striatum.

and Wei et $\mathrm{al}^{95}$ for resting state, and Vasic et al ${ }^{61}$ for taskrelated); others were speaking of "altered" or "pathological" connectivity only without specifying if the connection was hyper- or hypoconnected (Yu et al, ${ }^{48}$ Zeng et al ${ }^{15}$ and Zeng et $\mathrm{al}^{65}$ for resting state); two studies did not compare unipolar
MDD to healthy controls but to bipolar MDD (Marchand et al, ${ }^{63}$ task-related), or compared treatment-responders to non-responders (Downar et al, ${ }^{22}$ resting state). The study by Zhang et $\mathrm{al}^{97}$ (resting state) investigated alterations in nodal centralities rather than the connectivity. 
Table 5 Studies on functional connectivity with EEG, MEG and SPECT

\begin{tabular}{|c|c|c|c|c|c|c|c|}
\hline Author & Year & Task & $\begin{array}{l}\text { Decreased } \\
\text { connectivity }\end{array}$ & Increased connectivity & $\begin{array}{l}\text { Sample } \\
\text { size }\end{array}$ & $\begin{array}{l}\text { Medication } \\
\text { status }\end{array}$ & $\begin{array}{l}\text { Mean } \\
\text { symptom } \\
\text { severity }\end{array}$ \\
\hline $\begin{array}{l}\text { Holmes and } \\
\text { Pizzagalli69 }\end{array}$ & 2008 & Stroop task & $\begin{array}{l}<\text { rACC-MePFC } \\
<\text { rACC-IDLPFC }\end{array}$ & & 20 & Naive & $\begin{array}{l}\text { BDI } \\
22.55 \\
\pm 9.23\end{array}$ \\
\hline Fingelkurts et a ${ }^{68}$ & 2007 & Rest & \multicolumn{2}{|c|}{$\begin{array}{l}\text { Comparison of long/short-range FC between } \\
\text { the hemispheres and correlation with symptoms } \\
\text { severity }\end{array}$} & 12 & Naive & $\begin{array}{l}\text { HDRS } \\
24 \\
\pm 4\end{array}$ \\
\hline Lee et $\mathrm{a}^{67}$ & 2011 & Rest & \multicolumn{2}{|c|}{$\begin{array}{c}>\text { Within right fronto-temporal network at } \\
\text { delta/theta-frequencies correlated with poorer } \\
\text { AD-treatment response }\end{array}$} & 108 & Medicated & $\begin{array}{l}\text { HDRS } \\
27.94\end{array}$ \\
\hline Leistritz et $\mathrm{al}^{70}$ & 2010 & $\begin{array}{l}\text { Pain } \\
\text { anticipation }\end{array}$ & $\begin{array}{l}<\text { Generalized } \\
\text { partial directed } \\
\text { coherence after } \\
\text { pain stimulation }\end{array}$ & & 18 & Mixed & $\begin{array}{l}\text { BDI } \\
29.4 \\
\pm 9.7\end{array}$ \\
\hline Leuchter et al ${ }^{66}$ & 2012 & Rest & & $\begin{array}{l}>\text { Alpha, theta, delta- } \\
\text { coherence in long-distance } \\
\text { connections between } \\
\text { frontopolar/temporal/ } \\
\text { parietooccipital regions } \\
>\text { Higher beta in DLPFC } \\
\text { and temporal regions }\end{array}$ & 121 & Naive & $\begin{array}{l}\text { HDRS } \\
21.9 \\
\pm 3.6\end{array}$ \\
\hline Lu et al ${ }^{104}$ & 2013 & $\begin{array}{l}\text { Emotional } \\
\text { faces }\end{array}$ & $<$ ACC-AMYG & & 20 & Naive & - \\
\hline Olbrich et al ${ }^{103}$ & 2014 & Rest & \multicolumn{2}{|c|}{$\begin{array}{l}\text { Before treatment: } \\
\text { > Alpha frequency: subPFC-IDLPFC, } \\
\text { subPFC-MePFC; after treatment: } \\
>\text { Beta-frequency: subPFC-rDLPFC }\end{array}$} & 60 & Naive & $\begin{array}{l}\text { HDRS } \\
19.8 \\
\pm 6.2\end{array}$ \\
\hline Shajahan et al ${ }^{43}$ & 2002 & $\begin{array}{l}\text { Verbal } \\
\text { fluency }\end{array}$ & \multicolumn{2}{|c|}{$\begin{array}{l}\text { After transcranial magnetic stimulation treatment: } \\
\qquad>\text { DLF-loop and limbic loop }\end{array}$} & 15 & Mixed & $\begin{array}{l}\text { HDRS } \\
25 \\
\pm 5.7\end{array}$ \\
\hline
\end{tabular}

Notes: $<$, decreased connectivity; $>$, increased connectivity (if the information was available in the original article). A small $r$ or $I$ in front of the regions indicates if the finding was on the left (I) or right ( $r$ ) hemisphere. The study by Lu et al ${ }^{104}$ used MEG; the study by Shajahan et a ${ }^{43}$ used SPECT. I, left, $r$, right (if provided by the original work). Abbreviations: ACC, anterior cingulate cortex; AD, antidepressants; AMYG, amygdala; BDI, Beck Depression Inventory; DLF, dorsolateral frontal; EEG, electroencephalography; HDRS, Hamilton Depression Rating Scale; PFC, prefrontal cortex; DLPFC, dorsolateral PFC; MEG, magneto-encephalography; MePFC, medial PFC; SPECT, single-photon emission computed tomography.

Table 6 Studies on effective connectivity

\begin{tabular}{|c|c|c|c|c|c|c|c|c|}
\hline Author & Year & Method & Task & $\begin{array}{l}\text { Decreased } \\
\text { connectivity }\end{array}$ & $\begin{array}{l}\text { Increased } \\
\text { connectivity }\end{array}$ & $\begin{array}{l}\text { Sample } \\
\text { size }\end{array}$ & $\begin{array}{l}\text { Medication } \\
\text { status }\end{array}$ & $\begin{array}{l}\text { Mean symptom } \\
\text { severity }\end{array}$ \\
\hline Almeida et $\mathrm{al}^{29}$ & 2009 & fMRI & $\begin{array}{l}\text { Emotional } \\
\text { faces }\end{array}$ & $<$ IOMPFC-IAMYG & & 16 & Mixed & $\begin{array}{l}\text { HDRS } \\
24.6\end{array}$ \\
\hline Leistritz et $\mathrm{al}^{73}$ & 2013 & EEG & $\begin{array}{l}\text { Pain } \\
\text { anticipation }\end{array}$ & & $\begin{array}{l}>N W \text {-redundancy } \\
\text { after pain-processing }\end{array}$ & 18 & Mixed & $\begin{array}{l}\text { BDI } \\
29.4 \\
(9.7)\end{array}$ \\
\hline Lu et $\mathrm{al}^{72}$ & 2012 & MEG & $\begin{array}{l}\text { Emotional } \\
\text { faces }\end{array}$ & $<$ DLPFC-AMYG & $\begin{array}{l}>\text { AMYG-ACC } \\
>\text { ACC-DLPFC }\end{array}$ & 20 & $\begin{array}{l}\text { Not } \\
\text { available }\end{array}$ & $\begin{array}{l}\text { HDRS not } \\
\text { available }\end{array}$ \\
\hline Schlösser et al ${ }^{71}$ & 2008 & fMRI & Stroop task & & $>$ dACC-rACC & 16 & Naive & $\begin{array}{l}\text { BDI } \\
27.1(10.5)\end{array}$ \\
\hline
\end{tabular}

Notes: $<$, decreased connectivity; $>$, increased connectivity (if the information was available in the original article). A small $r$ or I in front of the regions indicates if the finding was on the left $(I)$ or right $(r)$ hemisphere.

Abbreviations: ACC, anterior cingulate cortex; AMYG, amygdala; BDI, Beck Depression Inventory; dACC, dorsal ACC; DLPFC, dorsolateral prefrontal cortex; EEG, electro-encephalography; HDRS, Hamilton Depression Rating Scale; MEG, magneto-encephalography; rACC, rostral ACC; OMPFC, orbitomedial prefrontal cortex. 
In order to further analyze the results, we created matrices for both resting state and task-related fMRI studies. In these matrices, we inserted the number of studies that found a certain altered connectivity. Several studies reported on relatively small subregions, eg, differentiated in a seed-based analysis between the pregenual anterior cingulate cortex (ACC) and the subgenual ACC, both assigned to region 13 (cingulate cortex/limbic lobe) in our comprised anatomical categorization (see Table 1). If the study reported the same kind of altered connectivity (eg, increase) from both regions to a certain other region (eg, the insula), this connection was counted only once. If the study reported different kinds of altered connectivity from the seed regions to a certain other region, it was counted as two findings.

\section{Results}

This section reports on findings on SC, FC, and EC. The FC section is structured in fMRI-based findings with resting state activity and task-related activity and in EEG-based findings.

\section{Neuroanatomy and network structure (SC)}

Anatomical aberrations of the brain's structure and organization, eg, concerning the gray matter or white matter tracts, either may contribute to the etiology of MDD or may be a long-term consequence of it, or both. Reduced fractional anisotropy (FA; anisotropy of diffusion processes within the brain to reconstruct fiber tracts by imaging methods) and consequently altered white matter tract integrity could be found. MDD-specific alterations in anisotropy of the superior longitudinal fasciculus connecting the frontal, occipital, parietal and temporal lobes were reported. ${ }^{32}$ Another important tract is the uncinate fasciculus, which connects the anterior cingulate cortex, the amygdala, and the orbitofrontal cortex with the medial temporal lobe. ${ }^{33}$ It is involved in affective processing and memory. ${ }^{33}$ An increased integrity of this tract between the anterior cingulate cortex and the amygdala was found in adolescent and geriatric depression. ${ }^{34,35}$ As reported in a DTI study by Pacheco et al, a serotonin transporter gene promoter region polymorphism (5HTTLPR) is associated with altered fronto-limbic white matter pathways and an increased probability of the onset of the disorder. ${ }^{36}$

Reduced gray matter volume and glial cell reduction in the postcentral cortex, insula, parietal and occipital lobes, as well as lateral orbitofrontal, superior, and medial temporal cortices seem to be related with depression. ${ }^{1,12,32}$ The amygdala plays a crucial role in emotion processing and is closely connected with other parts of the limbic system; in consequence, it was at the focus of neuroanatomical investigation on depression. Microstructural changes like increased glial cell density and an augmented number of fibers linking the amygdala to other regions were found in depression, even after remission. ${ }^{1,37}$ Microstructural aberrations were also found in the medial prefrontal cortex and in the hippocampus. ${ }^{38}$ Especially the volume of the hippocampus seems to be reduced. ${ }^{39}$ Not only gray matter volume changes but also aberrant glial cell densities in MDD-specific cortico-limbic networks have been reported. ${ }^{6,40}$

Differences in white matter tracts between healthy controls and MDD patients were found in the limbic, frontal, and thalamic projection fibers as well as in some cortical connections. ${ }^{32}$ Fang et al reported that the strength of connections in the cortico-limbic network was increased in depressed individuals. ${ }^{12}$ More specific findings on the structural abnormalities of only one region could be extracted from a study of Arnold et al. ${ }^{1}$ The authors showed a decreased mean diffusion and increased FA in the amygdala. On a microstructural level, this might indicate a greater cell density and an increased number of fibers. Stronger structural connections in MDD patients concerned the amygdala, the hippocampus, the cerebellum, and the brain stem. The cell density of the medial prefrontal cortex was found to be reduced in the DTI study of Arnold et al. ${ }^{1}$ It has to be considered that the findings of Fang et al and Arnold et al were based on the examination of individuals remitted from MDD. The idea behind this type of research was to find a possible "anatomical scar" caused by depression. In addition to altered microstructures in the amygdala, reduced gray matter volume and glial cell reduction in the medial prefrontal cortex were found. ${ }^{1,12}$

Singh et al used graph-theoretical analysis methods to examine structural gray matter networks in depressed patients. ${ }^{25}$ Brain regions and voxels are represented by nodes and structural or FC by edges between the nodes. Structurally defined networks were based on characteristics like gray matter volume, cortical thickness, surface area, and white matter connections between gray matter regions. MDD is characterized by a decreased clustering of structural networks, fewer strong nodes (hubs) in typical regions like medial frontal or medial temporal areas, and an increased connectivity including the amygdala and the medial orbitofrontal gyrus. These findings were interpreted as a less efficient brain network organization on a structural basis in depressed individuals. ${ }^{25}$ Another region with abnormal structural integrity (less diffusion processes corresponding to less activity within the anatomical structures) is the anterior genus of the corpus callosum. ${ }^{74}$ 
To conclude: MDD-related structural changes concern gray matter densities of limbic (eg, amygdala and hippocampus) and frontal regions and the connectome, especially corticolimbic networks including amygdala, hippocampus, orbitofrontal and medial-prefrontal cortical structures, but also temporal, thalamic, and cerebellum structures.

\section{FC \\ fMRI-based findings}

The results on FC investigated by fMRI are summarized in the graphical analyses (Figure 1). Since FC refers to correlations, the functional connections between brain areas are not displayed as arrows but rather as undirected lines.

\section{Resting state fMRI}

Cao et $\mathrm{a}^{14}$ and Tahmasian et al ${ }^{45}$ reported disrupted resting state FC of the hippocampus in medication-naive MDD patients. The hippocampus as seed region exhibited an increased FC to the middle frontal gyrus, the inferior parietal cortex, and the cerebellum. On the other hand, a reduced FC from the hippocampus to the superior frontal gyrus, frontoinsula operculum, inferior frontal gyrus, medial frontal gyrus, middle frontal gyrus, and inferior parietal lobe was shown.

In four studies, the $\mathrm{FC}$ of the subgenual anterior cingulate cortex to other brain regions was evaluated. Decreased FC was reported from the subgenual anterior cingulate cortex to the supragenual anterior cingulate cortex, middle frontal cortex, superior frontal cortex, inferior frontal cortex, superior temporal gyrus, insula, and precuneus. . $4,34,46,47^{\text {Increased FC was }}$ found in the insula, amygdala, dorsomedial prefrontal cortex, and the middle frontal gyrus. Increased FC was also shown between the posterior cingulate cortex, posterior anterior cingulate cortex, and the dorsolateral prefrontal cortex, middle frontal gyrus, inferior frontal gyrus, and the insula. In contrast to this, $\mathrm{FC}$ from the posterior and the posterior anterior cingulate cortex to the caudate nucleus, thalamus, superior medial frontal gyrus, and superior frontal gyrus was reduced., $3,19,47$

Three publications referred to the cerebellum. Altered FC was measured from the cerebellum to the prefrontal cortex, superior frontal gyrus, inferior frontal gyrus, middle frontal gyrus, DMN, precuneus, fusiform gyrus, orbito-frontal cortex, superior temporal gyrus, lingual gyrus, and hippocampus. Increased FC is notified for the connections from the cerebellum to the lingual gyrus, middle occipital gyrus, fusiform gyrus, parahippocampal gyrus, temporal pole, middle frontal cortex, inferior temporal gyrus, superior temporal gyrus, DMN, medial prefrontal cortex, hippocampus, thalamus, and anterior cingulate cortex in MDD patients. ${ }^{13,19,48}$
Sheline et al, ${ }^{14}$ Ye et $a l,{ }^{50}$ and Fox et al ${ }^{49}$ identified decreased FC of the dorsolateral prefrontal cortex to the dorsomedial prefrontal cortex, anterior cingulate cortex, parahippocampal gyrus, thalamus, precentral gyrus, and subgenual cingulate cortex. In contrast, increased FC from the cerebellum to the parietal lobe was observed.

According to Guo et a ${ }^{19}$ and Yu et al ${ }^{48}$ in MDD patients the medial prefrontal cortex is less functionally connected to the middle frontal gyrus, the superior frontal gyrus, and the anterior cingulate cortex, but closer connected to the precuneus, the thalamus, and the inferior temporal gyrus.

Within the DMN, the thalamus was more intensely connected to the anterior cingulate cortex and to the hippocampus. ${ }^{48,51}$ Two studies ${ }^{14,19}$ revealed a reduced FC between the precuneus of MDD patients and the thalamus and the superior medial frontal gyrus. A strengthened connectivity was described between the precuneus and the dorsomedial prefrontal cortex. Tahmasian et $\mathrm{a}^{45}$ found reduced FC strengths of the amygdala to the medial frontal gyrus, superior frontal gyrus, fronto insula, operculum, inferior frontal gyrus, and the inferior parietal lobe in MDD patients. Only one study casts light on the FC of the subgenual cingulate cortex to other brain regions, with evidence for an increased FC to the DMN. ${ }^{51}$

To complete the findings on the resting-state fMRI studies included in our graphic analysis, the data reported by Furman et $\mathrm{al}^{52}$ should be mentioned. This study found stronger FC in depressed patients from the ventral striatum to the precuneus, posterior cingulate cortex, cerebellum, inferior parietal lobe, from the dorsal caudate to the middle frontal gyrus, from the ventral rostral putamen to the caudate body, and from the dorsal caudate putamen to the inferior parietal lobe and the lingual gyrus. Weakened FC is described from the ventral striatum to the subgenual anterior cingulate cortex and the medial orbitofrontal cortex, from the dorsal caudate to the medial frontal gyrus, from the ventral rostral putamen to the superior temporal gyrus, the medial frontal gyrus, the orbitofrontal cortex and the posterior insula, from the dorsal caudate putamen to the superior temporal gyrus, middle temporal gyrus, and the posterior insula. ${ }^{52}$

Zeng et a ${ }^{65}$ used a machine-learning approach (clustering analysis) to automatically parcellate the perigenual anterior cingulate cortex into a subgenual and pregenual part, which were then used as seed areas for FC analysis. Stronger functional connections were found from the seeds to the following regions in MDD patients: ventrolateral prefrontal cortex, ventromedial prefrontal cortex, superior temporal gyrus, lentiform nucleus, hippocampus, insula, thalamus, superior 
A

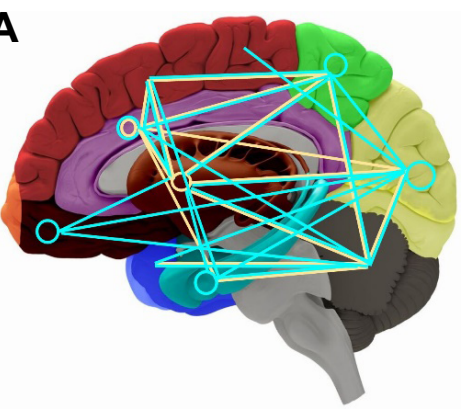

B

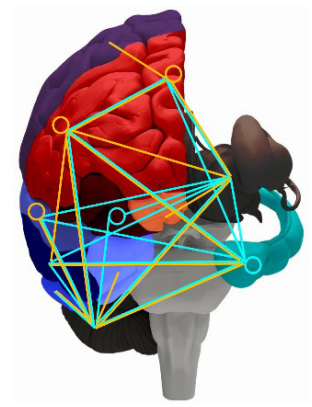

C

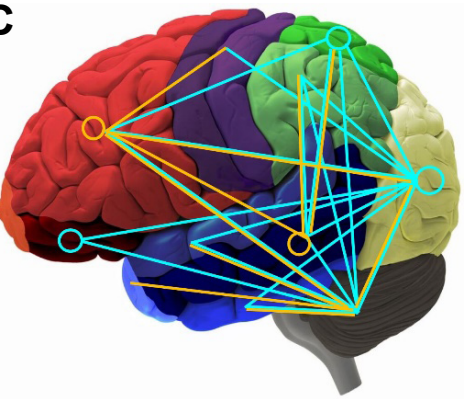

D
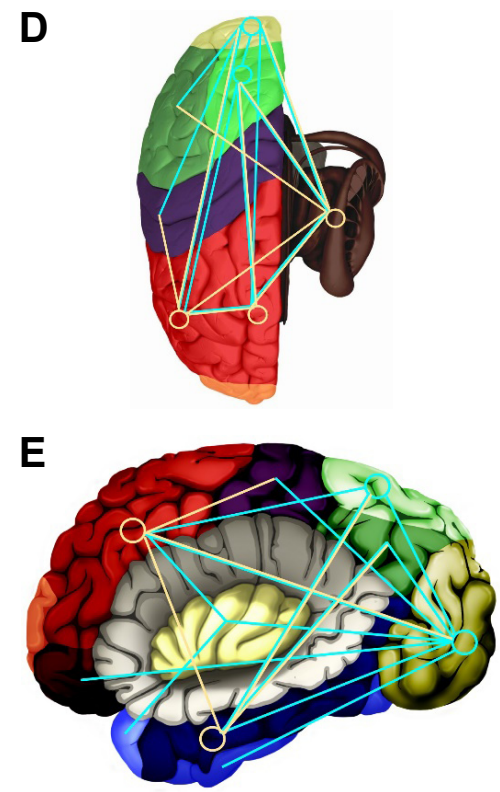

F

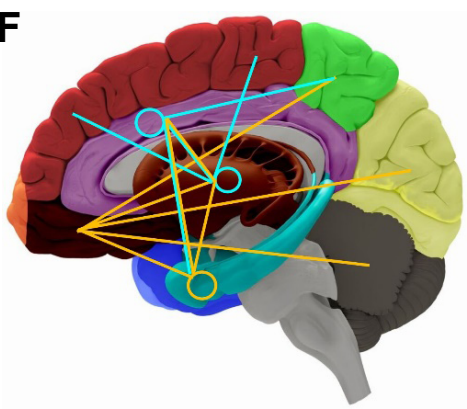

G

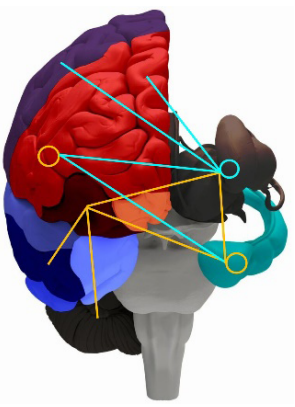

H

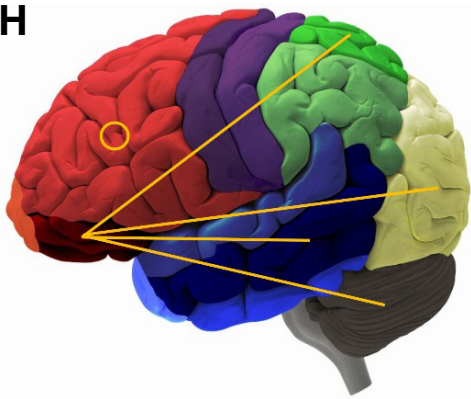

I
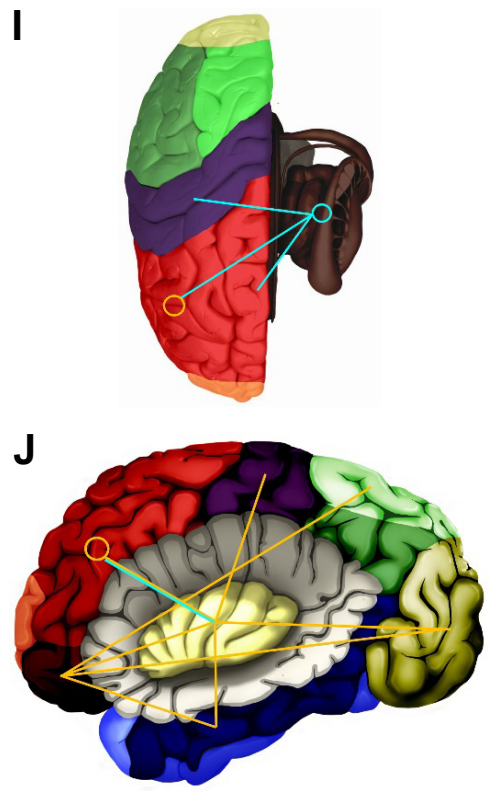

Figure I Functional connectivity (FC) for MDD patients compared with healthy controls for resting state (left) and task-related fMRI studies (right).

Notes: A/F: sagittal, lateral view; b/g: sagittal, anterior view; C/H: lateral view of the surface; D/I: sagittal, dorsal view; E/J: lateral view, insula displayed by removing the opercular brain segments. The orange lines represent increased, the blue lines decreased FC. The circles indicate an altered self-referential connectivity (ie, within the region). Colors of the brain regions are coded according to Table I.

Abbreviations: $\mathrm{FMRI}$, functional magnetic resonance imaging; MDD, major depressive disorder. 
frontal gyrus, amygdala, medial prefrontal cortex, precuneus/ posterior cingulate cortex (PCC), superior frontal gyrus, orbitofrontal cortex, middle temporal gyrus, superior temporal gyrus, angular gyrus, inferior temporal gyrus, supramarginal gyrus, and putamen. With this approach, the authors were able to reliably differentiate between patients and healthy controls based on differences in the FC. For the subgenual ACC connectivity, the correct identification was $92.5 \%$, and for the pregenual ACC $84.9 \%$.

Resuming the fMRI-based findings on resting-state connectivity in MDD, it should be noted that it is not a specific brain area, or even a small number of areas, which is involved in processing mood-related activity (see Figures 2 and 3). On the contrary, it seems to be a dys-connectivity of many regions, finally of the full brain, which creates mood disorders. As can be expected, frontal, prefrontal, and limbic structures are involved, but also basal ganglia, temporal structures, cerebellum, and the brain stem. Additionally, there seems no distinct over- or under-connectivity of the included networks, which may indicate state-dependent and context-related connectivity strengths. During resting states, brain activity may strongly depend on the specific thoughts and emotions which are experienced by an individual.

\section{Task-related fMRI}

From the 14 task-related fMRI studies, 10 were included in the graphic analyses, see Figure 1. The study of Frodl et a ${ }^{53}$ used the orbitofrontal cortex as a seed region during a facematching task and showed increased FC to the precuneus, cerebellum, middle cingulum, superior occipital gyrus, gyrus cerebelli, middle temporal gyrus, superior parietal gyrus, and the thalamus. Two other studies assessed the FC alterations of the subgenual anterior cingulate cortex in an emotional face paradigm and an executive control task. Increased FC was found between the subgenual anterior cingulate cortex, the amygdala, and the ventral striatum, reduced FC between the subgenual anterior cingulate cortex and the fusiform gyrus,

\begin{tabular}{|l|l|l|l|l|l|l|l|l|l|l|l|l|l|l|l|l|l|}
\hline & 1 & 2 & 3 & 4 & 5 & 6 & 7 & 8 & 9 & 10 & 11 & 12 & 13 & 14 & 15 & 16 & 17 \\
\hline 1 & & & & & & & & & & & & & & & & \\
\hline 2 & & 1 & & & & & & & & & & & & & & & \\
\hline 3 & & & 3 & 1 & 1 & & & & & 1 & & 2 & 4 & 3 & & 1 & 1 \\
\hline 4 & & & 1 & 1 & & 1 & & & & & & & 3 & 1 & & & \\
\hline 5 & & & & & & & & & & & & & & & & & \\
\hline 6 & & & 2 & 2 & & 1 & & & & 1 & & & & 1 & & & \\
\hline 7 & & & & & & & & & & 1 & & 1 & & 1 & & & \\
\hline 8 & & & & & & & & & & & & & & & & & 1 \\
\hline 9 & & & & & & & & & & & & & & & & & 1 \\
\hline 10 & & & & & & 1 & 1 & & & 1 & & 2 & & & & & \\
\hline 11 & & & & & & & & & & & & & & & & & 1 \\
\hline 12 & & & 2 & 1 & & & 1 & & 1 & 2 & & 1 & 1 & & & & 2 \\
\hline 13 & & & 6 & 4 & & 3 & 1 & & 1 & 1 & & & 4 & 2 & 2 & 2 & \\
\hline 14 & & 1 & & 2 & & 2 & & & 1 & 1 & & 1 & 4 & 1 & 1 & 1 \\
\hline 15 & & & 1 & & & & & 1 & 1 & & & 2 & 1 & 3 & & \\
\hline 16 & & 1 & 1 & & 1 & 1 & 1 & & 1 & 2 & 1 & 1 & & 1 & 2 & 2 & 1 \\
\hline 17 & & 1 & 2 & & & 2 & 1 & & 2 & 1 & 1 & 2 & 1 & 1 & & 1 & $\vdots$ \\
\hline
\end{tabular}

Figure 2 Synopsis of findings on FC in MDD compared to healthy controls using fMRI/resting state.

Notes: The matrix shows the findings on connectivity alterations between the 17 brain regions (see Table I), separated for decreased connectivity (lower triangle, blue) and increased connectivity (upper triangle, orange). The numbers in the cells specify the number of studies where this kind of altered connectivity was found.

Abbreviations: FC, functional connectivity; fMRI, functional magnetic resonance imaging; MDD, major depressive disorder. 


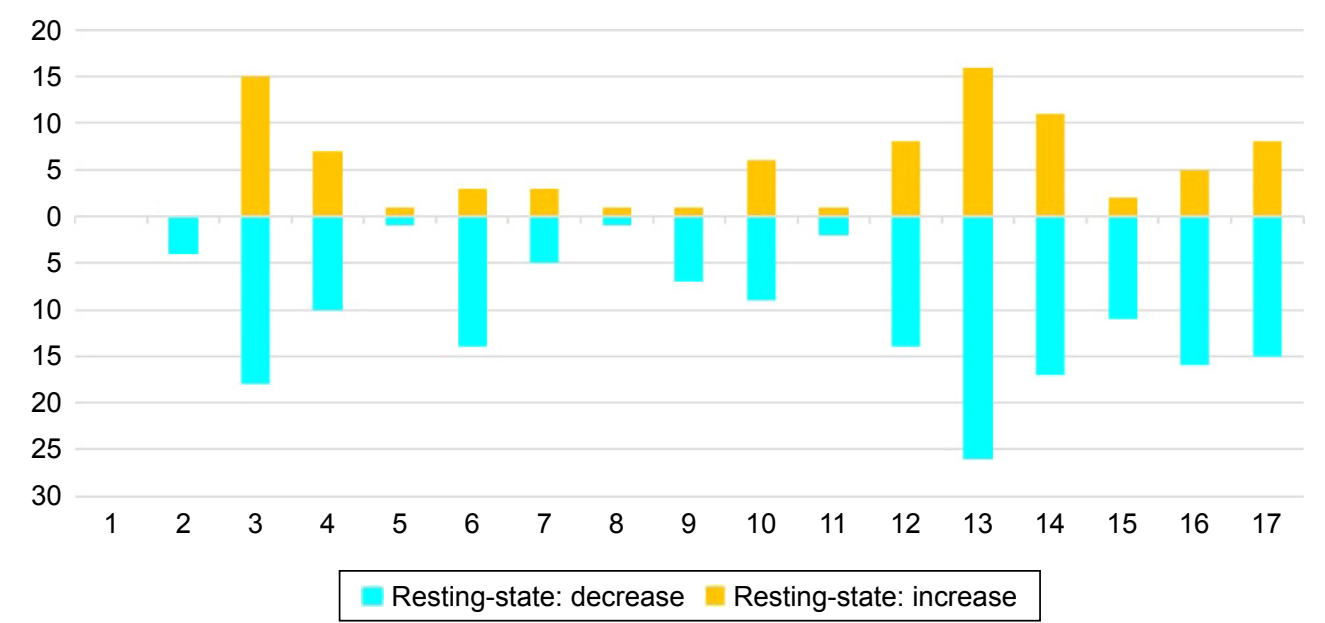

Figure 3 Histogram of the 17 regions found in $\mathrm{MMRI} /$ resting state studies showing the number of studies that found a region to have an altered FC with another region. Notes: Blue: decreased connectivities in MDD compared to healthy controls; orange: increased connectivities in MDD compared to healthy controls. Abbreviations: FC, functional connectivity; fMRI, functional magnetic resonance imaging; MDD, major depressive disorder.

precuneus, insula, middle frontal gyrus, cingulate, superior, and middle temporal gyrus. ${ }^{54,55}$

A reduced rostral prefrontal cortex-amygdala connectivity during fear conditions was described by Kong et al. ${ }^{100}$ In the work of Avery et al, ${ }^{56}$ the mid-dorsal insular cortex was used as a seed region, revealing increased FC to the middle temporal gyrus, amygdala, middle occipital gyrus, medial orbitofrontal cortex, cerebellum, and the precentral gyrus. In this study, a visceral interoceptive sensation task was used.

Marchand et $\mathrm{al}^{57}$ defined the precuneus and the thalamus as seeds and found FCs to be degraded to the inferior frontal gyrus, the supplementary motor area, the anterior cingulate cortex, the primary sensory motor cortex, and the thalamus in MDD patients when an executive control task was applied.

Applying an emotional picture paradigm or a personality trait task, the amygdala seems to be more strongly connected to the hippocampus and to the caudate-putamen, and the middle frontal gyrus seems to be more strongly connected to the dorsal anterior cingulate cortex and to the dorsolateral prefrontal cortex.$^{58,59}$ Strigo et al ${ }^{102}$ found the dorsal insular cortex more intensely connected to the posterior thalamus and lower connected to the inferior frontal gyrus under a pain anticipation task paradigm. In the study of van Tol et al, ${ }^{60}$ the aberrant connectivity ensuing from the DMN was found under an emotional face classification task. All connections to the orbitofrontal cortex, nucleus accumbens, medial prefrontal gyrus, frontal pole, the ventral caudate nucleus, the subgenual anterior cingulate cortex, and the putamen proved to be decreased in MDD individuals. ${ }^{60}$ Vasic et $\mathrm{l}^{61}$ found reduced $\mathrm{FC}$ in inferior parietal regions, superior prefrontal regions, fronto-polar regions, and increased FC in the dorsolateral and ventrolateral prefrontal cortex, cerebellum, anterior cingulate cortex, and the superior prefrontal cortex in MDD patients tested with working memory tasks.

One study investigated $\mathrm{FC}$ in both resting state and task-condition. ${ }^{64}$ With the anterior cingulate cortex used as seed region, there were decreased correlations to the pallido-striatum and the medial thalamus compared to healthy controls for resting state, and for neutral and positive pictures alike. For pictures with negative valence, only the connection to the pallidum-striatum was significant.

As found in studies investigating neuronal resting state activity, also in fMRI studies using task-related stimulation, large cortical (frontal, prefrontal, limbic) and subcortical networks (eg, basal ganglia, as the pallido-striatum) were involved in MDD (see Figures 4 and 5). The concrete structures which could be identified depend on the seed regions (in seed-dependent studies), on the concrete tasks, and on other conditions.

\section{EEG-based findings}

FC changes were also examined with EEG at resting state conditions. Higher alpha-, theta-, and delta-coherences in long-distance connections within fronto-polar, temporal, and parieto-occipital regions, and higher beta-coherence in dorsolateral prefrontal and temporal regions were found by Leuchter et al in depressed patients. ${ }^{66}$ A poorer treatment outcome of antidepressant therapy was found to be associated with higher FC strength within a right fronto-opercular network at delta and theta frequencies. ${ }^{67}$ Fingelkurts et a ${ }^{68}$ 


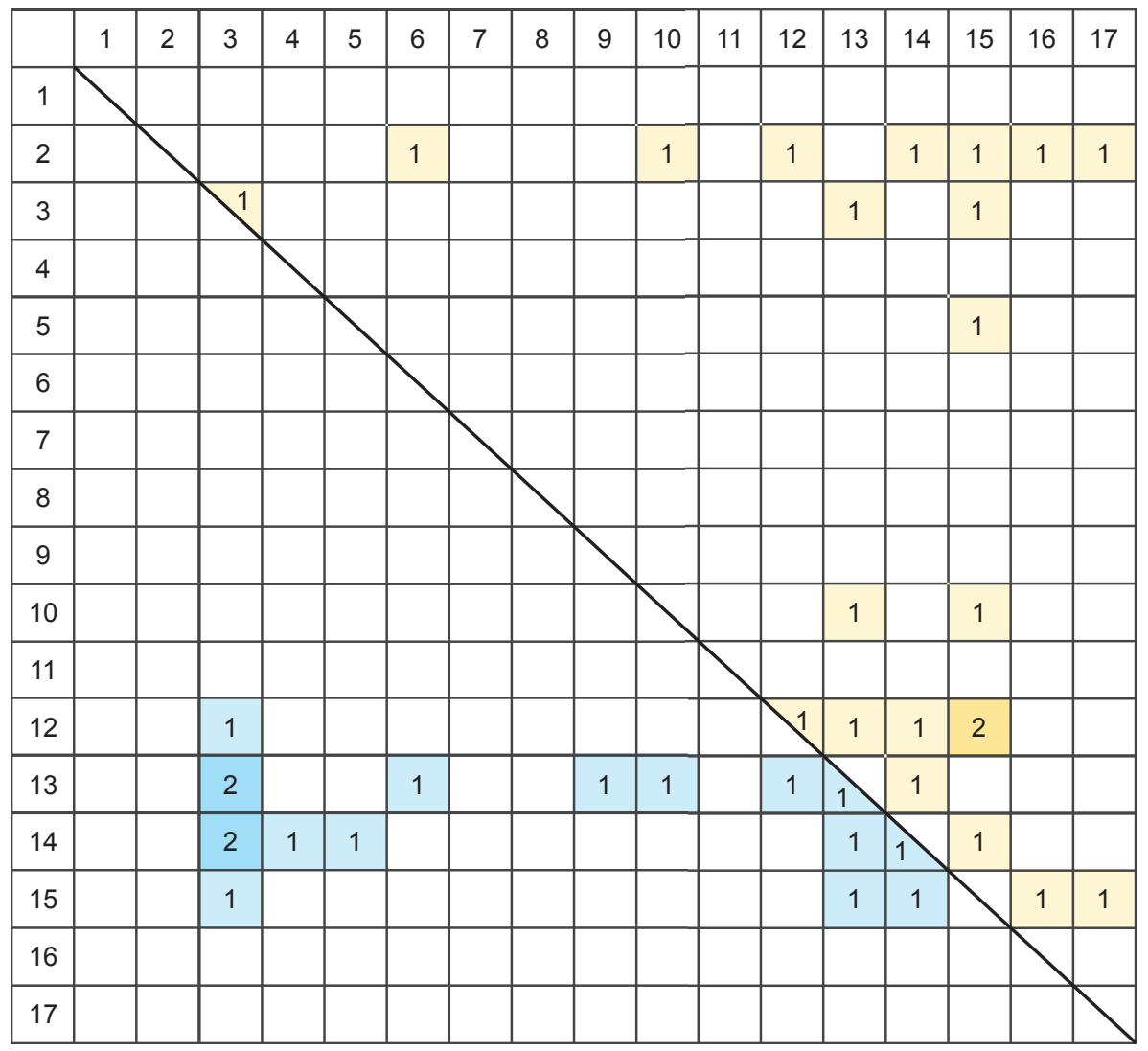

Figure 4 Synopsis of findings on functional connectivity in MDD compared to healthy controls using task-related fMRI.

Notes: The matrix shows the findings on FC alterations between the 17 brain regions (see Table I), separated for decreased connectivity (lower triangle, blue) and increased connectivity (upper triangle, orange). The numbers in the cells specify the number of studies where this kind of altered FC was found.

Abbreviations: FC, functional connectivity; fMRI, functional magnetic resonance imaging; MDD, major depressive disorder.

compared the strength of long- and short-range FC between the two hemispheres in relation to symptom severity and found that in depressive patients, the number and strength of short cortex functional connections were significantly more increased for the left than for the right hemisphere, while the number and strength of long functional connections were significantly larger for the right than for the left hemisphere.

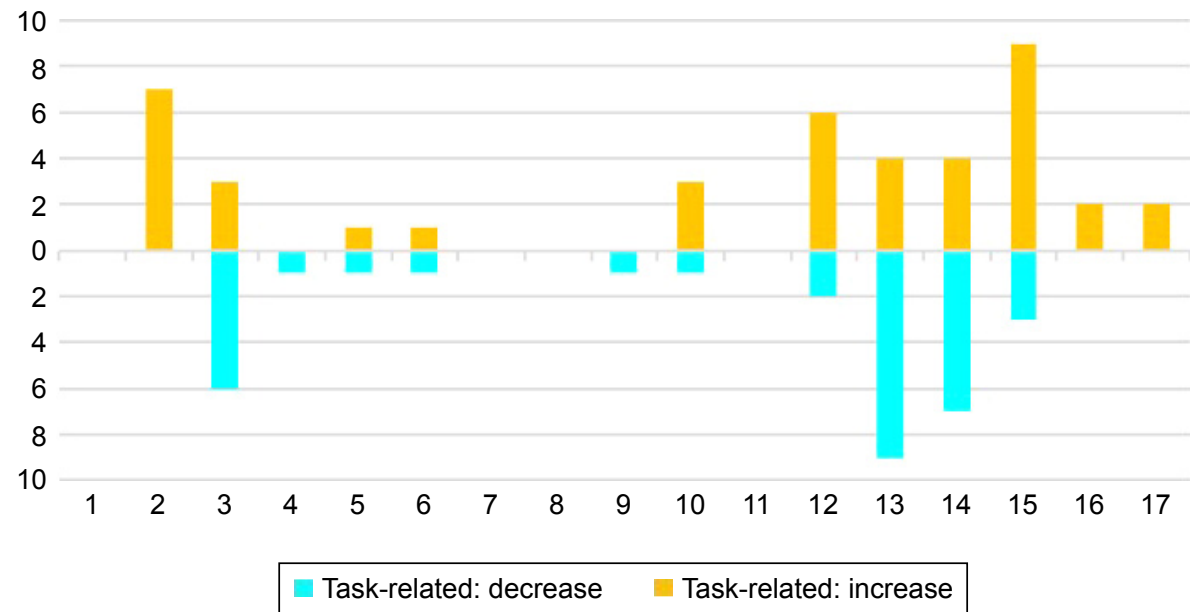

Figure 5 Histogram of the 17 regions found in $\mathrm{FMRI} /$ task-related studies showing the number of studies that found a region to have an altered FC with another region. Notes: Blue: decreased connectivities in MDD compared to healthy controls; orange: increased FC in MDD compared to healthy controls. Abbreviations: FC, functional connectivity; fMRI, functional magnetic resonance imaging; MDD, major depressive disorder. 
EEG was also utilized as an imaging method with both resting state and task-related recordings combined. A decreased connectivity between the anterior cingulate cortex and the medial as well as the dorsolateral prefrontal cortex was found by Holmes and Pizzagalli in depressed patients when tested with a Stroop task. ${ }^{69}$ Pain processing networks seem to be altered in depressed patients due to different FC. ${ }^{70}$

\section{Effective connectivity}

All studies on EC used a task-related design. Schlösser et a ${ }^{71}$ investigated alterations of EC in MDD patients compared to healthy controls using fMRI. They found reduced EC between the dorsal anterior and the rostral anterior cingulate cortex. Another study reported a reduced coupling between the dorsolateral prefrontal cortex and the amygdala as well as stronger connections between the anterior cingulate cortex, the amygdala, and the dorsolateral prefrontal cortex. ${ }^{72}$ Almeida et $\mathrm{al}^{29}$ assessed the EC between the amygdala and the orbitomedial prefrontal cortex during experiments with happy and sad emotional stimuli and found altered EC compared to healthy controls. Leistritz et $\mathrm{a}^{13}$ found reductions in the EC in depressed subjects and a reduced network redundancy in effective networks during pain processing.

\section{Discussion}

The functioning of the brain is based on network dynamics, including hierarchies of (sub-)networks and the interconnection of networks. Current imaging technologies open the way for measuring and reconstructing these networks and their dynamics. Compared to former decades when the understanding of brain functions was almost exclusively dependent on the study of brain lesions, now different imaging methods for the identification and analysis of connectivity in the living brain are available. ${ }^{41,42}$ In most of the imaging methods, the first step before any further analysis or modeling is the identification of nodes (brain regions). Connectivity can be defined on large scales (whole brain analysis) or on small or micro-scales, focusing on (sub-) populations of neurons. SC (the connectome) is assessed by DTI with FA or white matter tractography (WMT), based on the technique of MRI. DTI measures the diffusion of water molecules as a marker of cell or fiber density, with the directionality of the greatest diffusion coefficient indicating the pathway of the fibers. ${ }^{1}$ The analysis of static or dynamic FC is based on data from almost all available brain imaging technologies: fMRI, EEG, MEG, positron emission tomog- raphy (PET) or SPECT. ${ }^{26,43}$ Studies on EC usually are based on task-related fMRI-recordings and analyzed by structural equation modeling (SEM), dynamic causal modeling (DCM), or Granger causality mapping (GCM).

The number of studies on neuronal connectivity in MDD increased sharply during the last couple of years. This systematic review is a first provisional appraisal on the state of our knowledge and intends to provide a preliminary orientation on the findings. Taken together, the variance and inconsistency of the results are salient. Within the diversity of methods and findings, SC studies afford a more homogenous picture than studies on FC or EC. Evidently, SC is more stable than, eg, FC, less dynamic with reference to time and to changes in activity-dependent network recruitment, and easier to detect by the existing imaging methods.

Referring to FC, there are several reasons for varying findings and the inconsistency of the picture:

1. It matters how FC and EC are defined, and consequently what kind of analysis or modeling methods are used.

2. As FC is a correlative phenomenon based on the synchronization of brain areas, it does not imply any kind of directionality or causal relationship between the activities of the involved brain areas. FC is not a proof of a direct relation because synchronized activities may be mediated by additional structures or external sources, and it is not easy to clarify if functional connections to external sites are changed.

3. Connectivity is changing and dynamically varying (nonstationarity). During these dynamics, different networks are involved and the structure under consideration is modified by varying inputs affecting its activity and connectivity. In this case, dynamic full brain models as created by the FC dynamics (FCD) approach ${ }^{75}$ are of great importance for future research.

4. Many FC studies are performed with task-related recordings. The heterogeneity of the tasks and of the stimulation paradigms used has a great impact on neuronal connectivity, concerning direction, intensities, or network recruitment. For example, stimulation can be emotion-related or not emotion-related. Subjects are exposed to visual, verbal, or even acoustic stimuli and have only to perceive the stimulation or to react by fulfilling some executive tasks. (Re-)actions and decisions may be affected by more or less MDD-specific cognitive or motivational impairments..$^{20}$ Concerning studies on $\mathrm{EC}$, the problem might be even more distinct. This kind of connectivity is also dynamic and activity-dependent, 
which limits the selection of appropriate analysis methods. $^{26}$

5. Another source of diversity is the methods of data acquisition. Different imaging methods create different results. In the future, the combination of different methods like fMRI and EEG might be a key for creating a more comprehensive picture on connectivity dynamics and for validating findings from only one method.

6. Inconsistent results may be created by differences in medication and treatment. Some patients were medicated at the moment of measurement, others were washed-out after a period of drug intake and others were completely medication-free. Indeed, medication has an impact on neuronal connectivity. ${ }^{8,9}$

The results of the review are now discussed for all relevant brain regions.

An intensified neuronal connectivity of the medial temporal lobe to other brain regions during task-related recordings in MDD was demonstrated in our review. Connections of the frontal lobe, including the medial and the lateral frontal lobe, and the frontal pole to other brain regions are reduced during task-related conditions. The medial temporal lobe and the frontal lobe seem to be functionally and structurally altered in depression. ${ }^{25,27}$

An enlarged volume and an intensified activation of the amygdala may contribute to the pathogenesis of MDD, which is normalized during successful antidepressant treatment. ${ }^{43-45,76-78}$ The amygdala is crucially involved in the neuronal circuits mediating emotion perception and mood regulation. A decreased connectivity of the frontal lobe to limbic areas, eg, the amygdala or the cingulate cortex, activates the amygdala which in turn changes the emotional response to external or internal stimuli. Also, the orbitomedial prefrontal cortex (OMPFC) is involved in emotion regulation. Almeida et $\mathrm{al}^{29}$ investigated the abnormal right-left asymmetry in the OMPFC-amygdala anatomic connectivity and its role in the pathophysiology of emotion dysregulation, related to abnormal left vs right hemisphere processing of emotions. Happy and sad emotion labeling experiments investigated the EC and FC between the OMPFC and the amygdala in both hemispheres. MDD patients showed a significantly greater negative left-sided top-down OMPFC-to-amygdala EC than healthy persons in an experiment presenting happy stimuli. This increased amygdala inhibition to happy stimuli might be a neuronal basis of the intensified negative and reduced positive emotional perception biases in MDD. The findings support the hypothesis of a left-hemisphere dominance in the processing of positive emotions. Sad emotion labeling experiments revealed a decreased left-sided top-down OMPFC-to-amygdala EC in the processing of negative emotions in MDD patients. ${ }^{29,40}$ The data suggest a decreased regulatory effect of frontal regions onto limbic regions.

Not only the connection of the amygdala to fronto-cortical regions seems to be affected in MDD but also the connection of the frontal cortex to brain areas involved in memory processing. Positive or negative emotional arousal improves memory activities in healthy brains, ${ }^{78-80}$ whereas in MDD, the memory efficiency following negative stimuli is enhanced compared to healthy controls. The degree of over-activation of the neuronal memory system correlates with the severity of depressive symptoms. Depressed patients tend to have more negative memory biases and over-activate neuronal systems responding to negative affective contents. This could be explained by an increased connectivity between the amygdala, the hippocampus, and the caudate-putamen system as detected by Arnold et $\mathrm{al}^{1}$ during negative picture encoding tasks. Their experiments revealed an increased amygdala-centered fronto-limbic connectivity. ${ }^{1,21,58,81}$

By the DTI method, altered white matter tracts originating from the amygdala can be visualized. A hyperconnectivity to the amygdala seems to be a consistent and persistent MDDspecific finding, recognizable also in remitted MDD patients. In healthy controls, there is an increased functional resting state connectivity between the amygdala and the brainstem triggered by mild psychological stress. ${ }^{1}$ Microstructural alterations of the amygdala combined with activation and connectivity to other brain regions may cause vulnerability for depressive episodes. After a first depressive episode, there is a higher probability of developing further ones, with structural and functional effects consequential to each episode. ${ }^{1}$ Another component of vulnerability might be a generally diminished prefrontal activation in response to a challenge which does not disappear with medication and remission of symptoms. ${ }^{82}$

According to our review, the limbic cortex is less connected to other brain regions in MDD. Within this system, the subgenual anterior cingulate cortex is crucial in affective modulation, and its altered connectivity might contribute to uncontrolled emotional responses. ${ }^{46}$

The cerebellum is less connected to other brain regions both in resting state and in task-related recordings. In the past, it was assumed that the main function of the cerebellum is to control motor coordination. Recent studies showed that it is 
also involved in emotional and cognitive processes. ${ }^{19}$ Reduced cerebellar volume was found in brains of MDD patients. ${ }^{39}$

Neuronal connections to the insula are intensified in MDD. The insula is involved in interoceptive awareness and perception, which is closely related to emotion processing and decision making. ${ }^{84}$ Therefore the function of interoception-associated brain regions, eg, the insula, may be related to depressive symptoms. As the insula cooperates with the vagus nerve, stimulating this nerve might be a future opportunity for treatment. ${ }^{56}$

The basal ganglia system seems also to be involved in the neuronal network dynamics of MDD. The basal ganglia system is partially overlapping with limbic regions, eg, in the thalamus and the pallido-striatum. In mood disorders, the activity and the connections of both regions to other brain regions are enhanced and contribute to the aberrant corticolimbic mood-regulating circuits described above.

Concerning the DMN and other intrinsic networks (IC) that are commonly investigated in resting state connectivity studies on MDD, our results neither support the hypothesis of a dominance of the DMN over other IC, nor the hypothesis of a dysfunction of the salience network as the mediator between the DMN and the central executive network (CEN). On the contrary, our analysis reveals an overall decreased connectivity compared to healthy controls within the DMN (regions 4, 6, and 13 in our categorization), within the SN (regions 3, 13, and 15) and within the CEN (regions 2, 3, 4, and 7). Notably, no altered connectivity was found in any study within the CEN during the performance of tasks.

None of the studies in our review addressed gender differences, even though there is emerging evidence for distinct neuronal substrates between women and men, eg, in gray matter volumes ${ }^{112,113}$ or resting-state activity. ${ }^{114}$ Since this might be another reason for the diverging results in connectivity analyses, this topic should be taken into account in future studies. Last but not least, the investigation of interhemispheric connectivity with methods like the voxel-mirrored homotopic connectivity (VMHC, see Guo et $\mathrm{al}^{19}$ ) could contribute to understanding the heterogenic results of the studies.

\section{Conclusion}

This systematic review of MDD-specific neuronal connectivity reveals tabular and graphic synopses of studies published from 2002 to 2015. Aberrant neuronal connectivity concerns the medial-, orbito, and lateral-frontal lobe, frontal pole, limbic structures, cerebellum, medial-temporal lobe, basal ganglia, occipital lobe, and the insula. During resting state conditions, the FC of the medial frontal lobe, medial temporal lobe, and basal ganglia to other regions of the brain is increased, whereas the cerebellum is less connected with other brain regions. During task-related conditions, the limbic structures, medial- and lateral-frontal lobe, occipital lobe, cerebellum, and frontal pole are less connected to other brain regions in depressed compared with healthy people. Contrary to this, the medial temporal lobe, the orbitofrontal lobe, and the insula are stronger connected with many other brain regions.

Future research in MDD should focus on networks including the orbitomedial prefrontal cortex, the anterior cingulate cortex, the amygdala, the hippocampus, the insula, the cerebellum, and the basal ganglia as regions of interest. All these areas were repeatedly found to be involved in MDD-specific neuronal connectivity. Another important development is the measurement, analysis and mathematical modeling of network dynamics.

To reduce the variance of the results, experimental conditions should be unified regarding the stimulation, the subjects, and the imaging methods. Also, the regions of interest and the methods for modeling connectivity should become comparable. An interesting approach would be to combine different methods of data acquisition like EEG and fMRI to obtain a more converging and valid picture of altered connectivity. Since alterations in neuronal connectivity contribute to the etiology and to the symptoms of MDD, the modulation of neuronal networks may help in the development of effective and sustainable treatments.

\section{Author contributions}

All authors contributed toward data analysis, drafting and revising the paper and agree to be accountable for all aspects of the work.

\section{Disclosure}

The authors report no conflicts of interest in this work.

\section{References}

1. Arnold JF, Zwiers MP, Fitzgerald DA, et al. Fronto-limbic microstructure and structural connectivity in remission from major depression. Psychiatry Res. 2012;204(1):40-48.

2. Guo W, Liu F, Xue Z, et al. Decreased interhemispheric coordination in treatment-resistant depression: a resting-state FMRI study. PLoS One. 2013;8(8):e71368.

3. Horn DI, Yu C, Steiner J, et al. Glutamatergic and resting-state functional connectivity correlates of severity in major depression - the role of pregenual anterior cingulate cortex and anterior insula. Front Syst Neurosci. 2010;4:33.

4. Salomons TV, Dunlop K, Kennedy SH, et al. Resting-State CorticoThalamic-Striatal Connectivity Predicts Response to Dorsomedial Prefrontal rTMS in Major Depressive Disorder. Neuropsychopharmacology. 2014;39(2):488-498. 
5. Bear MF, Connors BW, Paradiso MA. Neuroscience: exploring the brain. 2nd ed. Baltimore: Lippincott, Williams \& Wilkins; 2001.

6. Leistedt SJ, Linkowski P. Brain, networks, depression, and more. Eur Neuropsychopharmacol. 2013;23(1):55-62.

7. Purves D, Williams SM. Neuroscience. 2nd ed. Sunderland, MS. Sinauer Associates. 2001.

8. Kozel FA, Rao U, Lu H, et al. Functional connectivity of brain structures correlates with treatment outcome in major depressive disorder. Front Psychiatry. 2011;2:7.

9. Li B, Liu L, Friston KJ, et al. A treatment-resistant default mode subnetwork in major depression. Biol Psychiatry. 2013;74(1):48-54.

10. Castrén E. Is mood chemistry? Nat Rev Neurosci. 2005;6(3):241-246.

11. Eickhoff SB, Grefkes C. Approaches for the Integrated Analysis of Structure, Function and Connectivity of the Human Brain. Clin EEG Neurosci. 2011;42(2):107-121.

12. Fang P, Zeng L-L, Shen H, et al. Increased cortical-limbic anatomical network connectivity in major depression revealed by diffusion tensor imaging. PLoS One. 2012;7(9):e45972.

13. Liu L, Zeng LL, Li Y, et al. Altered cerebellar functional connectivity with intrinsic connectivity networks in adults with major depressive disorder. PLoS One. 2012;7(6):e39516.

14. Sheline YI, Price JL, Yan Z, Mintun MA. Resting-state functional MRI in depression unmasks increased connectivity between networks via the dorsal nexus. Proc Natl Acad Sci U S A. 2010;107(24):11020-11025.

15. Zeng LL, Shen H, Liu L, et al. Identifying major depression using whole-brain functional connectivity: a multivariate pattern analysis Brain. 2012;135(Pt 5):1498-1507.

16. Kaiser RH, Andrews-Hanna JR, Wager TD, Pizzagalli DA. LargeScale Network Dysfunction in Major Depressive Disorder: A Metaanalysis of Resting-State Functional Connectivity. JAMA Psychiatry. 2015;72:603-611.

17. Chen Y, Wang C, Zhu X, Tan Y, Zhong Y, et al. Aberrant connectivity within the default mode network in first-episode, treatment-naïve major depressive disorder. $J$ Affect Disord. 2015;183:49-56.

18. Greicius MD, Krasnow B, Reiss AL, Menon V. Functional connectivity in the resting brain: A network analysis of the default mode hypothesis. Proceedings of the National Academy of Sciences. 2003;100(1):253-258.

19. Guo W, Liu F, Dai Y, et al. Decreased interhemispheric resting-state functional connectivity in first-episode, drug-naive major depressive disorder. Prog Neuropsychopharmacol Biol Psychiatry. 2013;41:24-29.

20. Schilbach L, Müller VI, Hoffstaedter F, et al. Meta-analytically informed network analysis of resting state FMRI reveals hyperconnectivity in an introspective socio-affective network in depression. PLoS One 2014;9(4):e94973.

21. Drevets WC, Price JL, Furey ML. Brain structural and functional abnormalities in mood disorders: implications for neurocircuitry models of depression. Brain Struct Funct. 2008;213(1-2):93-118.

22. Downar J, Geraci J, Salomons TV, et al. Anhedonia and RewardCircuit Connectivity Distinguish Nonresponders from Responders to Dorsomedial Prefrontal Repetitive Transcranial Magnetic Stimulation in Major Depression. Biol Psychiatry. 2014;76(3):176-185.

23. Vieussens R. Nevrographia Universalis, Lugundi: apud Joannem Certe, 1684

24. Fan Y, Shi F, Smith JK, Lin W, Gilmore JH, Shen D. Brain anatomical networks in early human brain development. Neuroimage. 2011;54(3): 1862-1871.

25. Singh MK, Kesler SR, Hadi Hosseini SM, et al. Anomalous Gray Matter Structural Networks in Major Depressive Disorder. Biol Psychiatry. 2013;74(10):777-785.

26. Friston KJ. Functional and Effective Connectivity: A Review. Brain Connect. 2011;1(1):13-36.

27. de Kwaasteniet B, Ruhe E, Caan M, et al. Relation between structural and functional connectivity in major depressive disorder. Biol Psychiatry. 2013;74(1):40-47.

28. Ma C, Ding J, Li J, et al. Resting-state functional connectivity bias of middle temporal gyrus and caudate with altered gray matter volume in major depression. PLoS One. 2012;7(9):e45263.
29. de Almeida JR, Versace A, Mechelli A, et al. Abnormal amygdalaprefrontal effective connectivity to happy faces differentiates bipolar from major depression. Biol Psychiatry. 2009;66(5):451-459.

30. Brodmann K, Gary LJ. Brodmann's Localization in the Cerebral Cortex: The Principles of Comparative Localisation in the Cerebral Cortex based on Cytoarchitectonics. New York, NY: Springer; 2006.

31. Miller K. Biomechanics of the Brain, Biological and Medical Physics, Biomedical Engineering. New York: Springer; 2011.

32. Korgaonkar MS, Cooper NJ, Williams LM, Grieve SM. Mapping inter-regional connectivity of the entire cortex to characterize major depressive disorder: a whole-brain diffusion tensor imaging tractography study. Neuroreport. 2012;23(9):566-571.

33. Papagno C. Naming and the Role of the Uncinate Fasciculus in Language Function. Curr Neurol Neurosci Rep. 2011;11(6):553-559.

34. Cullen KR, Gee DG, Klimes-Dougan B, et al. A preliminary study of functional connectivity in comorbid adolescent depression. Neurosci Lett. 2009;460(3):227-231.

35. Mettenburg JM, Benzinger TL, Shimony JS, Snyder AZ, Sheline YI. Diminished performance on neuropsychological testing in late life depression is correlated with microstructural white matter abnormalities. Neuroimage. 2012;60(4):2182-2190.

36. Pacheco J, Beevers CG, Benavides C, McGeary J, Stice E, Schnyer DM. Frontal-limbic white matter pathway associations with the serotonin transporter gene promoter region (5-HTTLPR) polymorphism. J Neurosci. 2009;29(19):6229-6233.

37. Erk S, Mikschl A, Stier S, et al. Acute and sustained effects of cognitive emotion regulation in major depression. $J$ Neurosci. 2010;30(47):15726-15734.

38. Ma N, Li L, Shu N, et al. White matter abnormalities in first-episode, treatment-naive young adults with major depressive disorder. $\mathrm{Am} \mathrm{J}$ Psychiatry. 2007;164(5):823-826.

39. Frodl T, Jager M, Smajstrlova I. Effect of hippocampal and amygdala volumes on clinical outcomes in major depression: a 3-year prospective magnetic resonance imaging study. $J$ Psychiatry Neurosci. 2008;33(5):423-430.

40. Bennett MR. The prefrontal-limbic network in depression: A core pathology of synapse regression. Prog Neurobiol. 2011;93(4):457-467.

41. Behrens TE, Sporns O. Human connectomics. Curr Opin Neurobiol. 2012;22(1):144-153.

42. Friston K. Dynamic causal modeling and Granger causality Comments on: The identification of interacting networks in the brain using fMRI: Model selection, causality and deconvolution. Neuroimage. 2011; 58(2-2):303.

43. Shajahan PM, Glabus MF, Steele JD, et al. Left dorsolateral repetitive transcranial magnetic stimulation affects cortical excitability and functional connectivity, but does not impair cognition in major depression. Prog Neuropsychopharmacol Biol Psychiatry. 2002;26(5):945-954.

44. Cao X, Liu Z, Xu C, et al. Disrupted resting-state functional connectivity of the hippocampus in medication-naïve patients with major depressive disorder. J Affect Disord. 2012;141(2-3):194-203.

45. Tahmasian M, Knight DC, Manoliu A, et al. Aberrant intrinsic connectivity of hippocampus and amygdala overlap in the fronto-insular and dorsomedial-prefrontal cortex in major depressive disorder. Front Hum Neurosci. 2013;7:639.

46. Connolly CG, Wu J, Ho TC, et al. Resting-state functional connectivity of subgenual anterior cingulate cortex in depressed adolescents. Biol Psychiatry. 2013;74(12):898-907.

47. Davey CG, Harrison BJ, Yücel M, Allen NB. Regionally specific alterations in functional connectivity of the anterior cingulate cortex in major depressive disorder. Psychol Med. 2012;42(10):2071-2081.

48. Yu Y, Shen H, Zeng LL, Ma Q, Hu D. Convergent and divergent functional connectivity patterns in schizophrenia and depression. PLoS One. 2013;8(7):e68250.

49. Fox MD, Buckner RL, White MP, Greicius MD, Pascual-Leone A. Efficacy of transcranial magnetic stimulation targets for depression is related to intrinsic functional connectivity with the subgenual cingulate. Biol Psychiatry. 2012;72(7):595-603. 
50. Ye T, Peng J, Nie B, et al. Altered functional connectivity of the dorsolateral prefrontal cortex in first-episode patients with major depressive disorder. Eur J Radiol. 2012;81(12):4035-4040.

51. Greicius MD, Flores BH, Menon V, et al. Resting-state functional connectivity in major depression: abnormally increased contributions from subgenual cingulate cortex and thalamus. Biol Psychiatry. 2007; 62(5):429-437.

52. Furman DJ, Hamilton JP, Gotlib IH. Frontostriatal functional connectivity in major depressive disorder. Biol Mood Anxiety Disord. 2011; 1(1): 11 .

53. Frodl T, Bokde AL, Scheuerecker J, et al. Functional connectivity bias of the orbitofrontal cortex in drug-free patients with major depression. Biol Psychiatry. 2010;67(2):161-167.

54. Davey CG, Yücel M, Allen NB, Harrison BJ. Task-related deactivation and functional connectivity of the subgenual cingulate cortex in major depressive disorder. Front Psychiatry. 2012;3:14.

55. Ho TC, Yang G, Wu J, et al. Functional connectivity of negative emotional processing in adolescent depression. J Affect Disord. 2014;15:65-74.

56. Avery JA, Drevets WC, Moseman SE, Bodurka J, Barcalow JC, Simmons WK. Major Depressive Disorder is associated with abnormal interoceptive activity and functional connectivity in the insula. Biol Psychiatry. 2014;76(3):258-266.

57. Marchand WR, Lee JN, Suchy Y, Johnson S, Thatcher J, Gale P. Aberrant functional connectivity of cortico-basal ganglia circuits in major depression. Neurosci Lett. 2012;514(1):86-90.

58. Hamilton JP, Gotlib IH. Neural substrates of increased memory sensitivity for negative stimuli in major depression. Biol Psychiatry. 2008;63(12):1155-1162.

59. Lemogne C, le Bastard G, Mayberg H, et al. In search of the depressive self: extended medial prefrontal network during self-referential processing in major depression. Soc Cogn Affect Neurosci. 2009;4(3): 305-312.

60. van Tol MJ, Veer IM, van der Wee NJ, et al. Whole-brain functional connectivity during emotional word classification in medication-free Major Depressive Disorder: Abnormal salience circuitry and relations to positive emotionality. Neuroimage. 2013;2:790-796.

61. Vasic N, Walter H, Sambataro F, Wolf RC. Aberrant functional connectivity of dorsolateral prefrontal and cingulate networks in patients with major depression during working memory processing. Psychol Med. 2009;39(6):977-987.

62. Heller AS, Johnstone T, Light SN, et al. Relationships between changes in sustained fronto-striatal connectivity and positive affect in major depression resulting from antidepressant treatment. Am J Psychiatry. 2013;170(2):197-206

63. Marchand WR, Lee JN, Johnson S, Gale P, Thatcher J. Differences in functional connectivity in major depression versus bipolar II depression. $J$ Affect Disord. 2013;150(2):527-532.

64. Anand A, Li Y, Wang Y, et al. Activity and connectivity of brain mood regulating circuit in depression: a functional magnetic resonance study. Biol Psychiatry. 2005;57(10):1079-1088.

65. Zeng LL, Shen H, Liu L, Hu D. Unsupervised classification of major depression using functional connectivity MRI. Hum Brain Mapp. 2014; 35(4):1630-1641.

66. Leuchter AF, Cook IA, Hunter AM, Cai C, Horvath S. Resting-state quantitative electroencephalography reveals increased neurophysiologic connectivity in depression. PLoS One. 2012;7(2):e32508.

67. Lee TW, Wu YT, Yu YW, Chen MC, Chen TJ. The implication of functional connectivity strength in predicting treatment response of major depressive disorder: A resting EEG study. Psychiatry Res. 2011; 194(3):372-377.

68. Fingelkurts AA, Fingelkurts AA, Rytsälä H, Suominen K, Isometsä E, Kähkönen S. Impaired functional connectivity at EEG alpha and theta frequency bands in major depression. Hum Brain Mapp. 2007;28(3): 247-261.

69. Holmes AJ, Pizzagalli DA. Spatiotemporal dynamics of error processing dysfunctions in major depressive disorder. Arch Gen Psychiatry. 2008;65(2):179-188.
70. Leistritz L, Weiss T, Ionov J, Bär KJ, Miltner WH, Witte H. Connectivity Analysis of Somatosensory Evoked Potentials in Patients with Major Depression. Methods Inf Med. 2010;49(5):484-491.

71. Schlösser RG, Wagner G, Koch K, Dahnke R, Reichenbach JR, Sauer $\mathrm{H}$. Fronto-cingulate effective connectivity in major depression: A study with fMRI and dynamic causal modeling. Neuroimage. 2008; 43(3):645-655.

72. Lu Q, Li H, Luo G, et al. Impaired prefrontal-amygdala effective connectivity is responsible for the dysfunction of emotion process in major depressive disorder: A dynamic causal modeling study on MEG. Neurosci Lett. 2012;523(2):125-130.

73. Leistritz L, Weiss T, Bär KJ, et al. Network Redundancy Analysis of Effective Brain Networks; a Comparison of Healthy Controls and Patients with Major Depression. PLoS One. 2013;8(4):e60956.

74. Xu K, Jiang W, Ren L, et al. Impaired interhemispheric connectivity in medicationnaive patients with major depressive disorder. J Psychiatry Neurosci. 2013;38(1):43-48.

75. Hansen EC, Battaglia D, Spiegler A, Deco G, Jirsa VK. Functional connectivity dynamics: Modeling the switching behavior of the resting state. Neuroimage. 2015;105:525-535.

76. Arnone D, McKie S, Elliott R, et al. Increased amygdala responses to sad but not fearful faces in major depression: relation to mood state and pharmacological treatment. Am J Psychiatry. 2012;169(8):841-850.

77. Frodl T, Meisenzahl EM, Zetzsche T, et al. Larger amygdala volumes in first depressive episode as compared to recurrent major depression and healthy control subjects. Biol Psychiatry. 2003;53(4):338-344.

78. Lange $\mathrm{C}$, Irle E. Enlarged amygdala volume and reduced hippocampal volume in young women with major depression. Psychol Med. 1999; 34(6):1059-1064.

79. Cahill L, McGaugh JL. Mechanisms of emotional arousal and lasting declarative memory. Trends Neurosci. 1998;21(7):294-299.

80. Dirnberger G, Hesselmann G, Roiser JP, Preminger S, Jahanshahi M, Paz R. Give it time: Neural evidence for distorted time perception and enhanced memory encoding in emotional situations. Neuroimage. 2012; 63(1):591-599.

81. Paz R, Pare D. Physiological basis for emotional modulation of memory circuits by the amygdala. Curr Opin Neurobiol. 2013;23(3):381-386.

82. Drevets WC. Prefrontal Cortical-Amygdalar Metabolism in Major Depression. Ann N Y Acad Sci. 1999;877:614-637.

83. Hooley JM, Gruber SA, Parker HA, Guillaumot J, Rogowska J, Yurgelun-Todd DA. Cortico-limbic response to personally challenging emotional stimuli after complete recovery from depression. Psychiatry Res. 2009;172(1):83-91.

84. Pollatos O, Gramann K, Schandry R. Neural systems connecting interoceptive awareness and feelings. Hum Brain Mapp. 2007;28(1): 9-18.

85. Abbott CC, Lemke NT, Gopal S, et al. Electroconvulsive therapy response in major depressive disorder: a pilot functional network connectivity resting state FMRI investigation. Front Psychiatry. 2013;4:10.

86. Guo W, Liu F, Xue Z, et al. Abnormal resting-state cerebellar-cerebral functional connectivity in treatment-resistant depression and treatment sensitive depression. Prog Neuropsychopharmacol Biol Psychiatry. 2013;44:51-57.

87. Guo W, Liu F, Xiao C, et al. Decreased insular connectivity in drugnaive major depressive disorder at rest. J Affect Disord. 2015;179: 31-37.

88. Lai CH, Wu YT. Decreased inter-hemispheric connectivity in anterior sub-network of default mode network and cerebellum: significant findings in major depressive disorder. Int J Neuropsychopharmacol. 2014;17(12):1935-1942.

89. Liu TY, Hsieh JC, Chen YS, Tu PC, Su TP, Chen LF. Different patterns of abnormal gamma oscillatory activity in unipolar and bipolar disorder patients during an implicit emotion task. Neuropsychologia. 2012;50(7):1514-1520.

90. Perrin JS, Merz S, Bennett DM, et al. Electroconvulsive therapy reduces frontal cortical connectivity in severe depressive disorder. Proc Natl Acad Sci U S A. 2012;109(14):5464-5468. 
91. Ramasubbu R, Konduru N, Cortese F, Bray S, Gaxiola-Valdez I, Goodyear B. Reduced intrinsic connectivity of amygdala in adults with major depressive disorder. Front Psychiatry. 2014;5:17.

92. Veer IM, Beckmann CF, van Tol MJ. Whole brain resting-state analysis reveals decreased functional connectivity in major depression. Front Syst Neurosci. 2010;4:41.

93. Wang L, Dai Z, Peng H, et al. Overlapping and segregated restingstate functional connectivity in patients with major depressive disorder with and without childhood neglect. Hum Brain Mapp. 2014;35(4): 1154-1166.

94. Wang L, Li K, Zhang QE, et al. Interhemispheric functional connectivity and its relationships with clinical characteristics in major depressive disorder: a resting state fMRI study. PLoS One. 2013;8(3): e60191.

95. Wei M, Qin J, Yan R, Li H, Yao Z, Lu Q. Identifying major depressive disorder using Hurst exponent of resting-state brain networks. Psychiatry Res. 2013;214(3):306-312.

96. Zhang YF, Han Y, Wang YZ, et al. Characterization of resting-state fMRI-derived functional connectivity in patients with deficiency versus excess patterns of major depression. Complement Ther Med. 2015;23(1):7-13.

97. Zhang J, Wang J, Wu Q, et al. Disrupted brain connectivity networks in drug-naive, first-episode major depressive disorder. Biol Psychiatry. 2011;70(4):334-342.

98. Zhu X, Wang X, Xiao J, et al. Evidence of a dissociation pattern in resting-state default mode network connectivity in first-episode, treatment-naive major depression patients. Biol Psychiatry. 2012;71(7): 611-617.

99. Henje Blom E, Connolly CG, Ho TC, et al. Altered insular activation and increased insular functional connectivity during sad and happy face processing in adolescent major depressive disorder. $J$ Affect Disord. 2015;178:215-223.

100. Kong L, Chen K, Tang Y, et al. Functional connectivity between the amygdala and prefrontal cortex in medication-naive individuals with major depressive disorder. J Psychiatry Neurosci. 2013;38(6):417-422.

101. Aertsen A, Preissl H. Dynamics of activity and connectivity in physiological neuronal networks. In: Schuster HG (ed.) Nonlinear Dynamics and Neuronal Networks. New York: VHC Publishers; 2001:281-302.

102. Strigo IA, Matthews SC, Simmons AN. Decreased frontal regulation during pain anticipation in unmedicated subjects with major depressive disorder. Transl Psychiatry. 2013;3(3):e239.
103. Olbrich S, Tränkner A, Chittka T, Hegerl U, Schönknecht P. Functional connectivity in major depression: Increased phase synchronization between frontal cortical EEG-source estimates. Psychiatry Res. 2014; 222(1-2):91-99.

104. Lu Q, Wang Y, Luo G, Li H, Yao Z. Dynamic connectivity laterality of the amygdala under negative stimulus in depression: A MEG study. Neurosci Lett. 2013;547:42-47.

105. Brakowski J, Spinelli S, Dörig N, et al. Resting state brain network function in major depression - Depression symptomatology, antidepressant treatment effects, future research. J Psychiatr Res. 2017;92: 147-159.

106. Mulders PC, van Eijndhoven PF, Schene AH, Beckmann CF, Tendolkar I. Resting-state functional connectivity in major depressive disorder: A review. Neurosci Biobehav Rev. 2015;56:330-344.

107. Hamilton JP, Farmer M, Fogelman P, Gotlib IH. Depressive Rumination, the Default-Mode Network, and the Dark Matter of Clinical Neuroscience. Biol Psychiatry. 2015;78(4):224-230.

108. Jiang J, Zhao YJ, Hu XY, et al. Microstructural brain abnormalities in medication-free patients with major depressive disorder: a systematic review and meta-analysis of diffusion tensor imaging. J Psychiatry Neurosci. 2017;42(3):150-163.

109. Kaiser RH, Andrews-Hanna JR, Wager TD, Pizzagalli DA. LargeScale Network Dysfunction in Major Depressive Disorder. JAMA Psychiatry. 2015;72(6):603-611.

110. Alamian G, Hincapié AS, Combrisson E, et al. Alterations of Intrinsic Brain Connectivity Patterns in Depression and Bipolar Disorders: A Critical Assessment of Magnetoencephalography-Based Evidence. Front Psychiatry. 2017;8:41.

111. Chen G, Hu X, Li L, et al. Disorganization of white matter architecture in major depressive disorder: a meta-analysis of diffusion tensor imaging with tract-based spatial statistics. Sci Rep. 2016;6:21825.

112. Carlson JM, Depetro E, Maxwell J, Harmon-Jones E, Hajcak G. Gender moderates the association between dorsal medial prefrontal cortex volume and depressive symptoms in a subclinical sample. Psychiatry Res. 2015;233(2):285-288.

113. Kong L, Chen K, Womer F, et al. Sex differences of gray matter morphology in cortico-limbic-striatal neural system in major depressive disorder. J Psychiatr Res. 2013;47(6):733-739.

114. Yao Z, Yan R, Wei M, Tang H, Qin J, Lu Q. Gender differences in brain activity and the relationship between brain activity and differences in prevalence rates between male and female major depressive disorder patients: a resting-state fMRI study. Clin Neurophysiol. 2014;125(11):2232-2239.
Neuropsychiatric Disease and Treatment

\section{Publish your work in this journal}

Neuropsychiatric Disease and Treatment is an international, peerreviewed journal of clinical therapeutics and pharmacology focusing on concise rapid reporting of clinical or pre-clinical studies on a range of neuropsychiatric and neurological disorders. This journal is indexed on PubMed Central, the 'PsycINFO' database and CAS,

\section{Dovepress}

and is the official journal of The International Neuropsychiatric Association (INA). The manuscript management system is completely online and includes a very quick and fair peer-review system, which is all easy to use. Visit http://www.dovepress.com/testimonials.php to read real quotes from published authors. 